OPEN ACCESS

Edited by:

Claudia Ruiz-Capillas, Instituto de Ciencia y Tecnología de Alimentos y Nutrición (ICTAN), Spain

Reviewed by: Tatiana Colombo Pimentel, Federal Institute of Education, Science and Technology of Paraná,

Brazil

Sergio Perez-Burillo, University of Granada, Spain

*Correspondence:

Kate Howell

khowell@unimelb.edu.au

Specialty section:

This article was submitted to

Food Microbiology,

a section of the journal

Frontiers in Microbiology

Received: 16 August 2021 Accepted: 28 October 2021 Published: 07 December 2021

Citation:

Chan M, Liu D, Wu Y, Yang F and Howell K (2021) Microorganisms in Whole Botanical Fermented Foods Survive Processing and Simulated Digestion to Affect Gut Microbiota

Composition.

Front. Microbiol. 12:759708. doi: 10.3389/fmicb.2021.759708

\section{Microorganisms in Whole Botanical Fermented Foods Survive Processing and Simulated Digestion to Affect Gut Microbiota Composition}

\author{
Miin Chan, Di Liu, Yingying Wu, Fan Yang and Kate Howell* \\ School of Agriculture and Food, The University of Melbourne, Parkville, VIC, Australia
}

Botanical fermented foods have been shown to improve human health, based on the activity of potentially beneficial lactic acid bacteria $(\angle A B)$ and yeasts and their metabolic outputs. However, few studies have explored the effects of prolonged storage and functional spices on microbial viability of whole fermented foods from fermentation to digestion. Even fewer have assessed their impact on the gut microbiota. Our study investigated the effects of production processes on $\angle A B$ and yeast microbial viability and gut microbiota composition. We achieved this by using physicochemical assessments and an in vitro gastrointestinal and a porcine gut microbiota model. In low-salt sauerkraut, we assessed the effects of salt concentration, starter cultures, and prolonged storage, and in tibicos, prolonged storage and the addition of spices cayenne, ginger, and turmeric. In both food matrices, LAB counts significantly increased $(p<0.05)$, reaching a peak of $7-8$ log cfu/g, declining to $6-6.5 \mathrm{log} c f u / g$ by day 96 . Yeast viability remained at 5-6 log $\mathrm{cfu} / \mathrm{g}$ in tibicos. Ginger tibicos had significantly increased $L A B$ and yeast viability during fermentation and storage $(p<0.05)$. For maximum microbial consumption, tibicos should be consumed within 28 days, and sauerkraut, 7 weeks. Simulated upper Gl digestion of both products resulted in high microbial survival rates of $70-80 \%$. The $82 \%$ microbial survival rate of cayenne tibicos was significantly higher than other treatments $(p<0.05)$. 16S rRNA sequencing of simulated porcine colonic microbiota showed that both spontaneously fermented sauerkraut and tibicos increase the relative abundance of Megasphaera 85-fold. These findings will inform researchers, producers, and consumers about the factors that affect the microbial content of fermented foods, and their potential effects on the gut.

Keywords: water kefir, tibicos, yeasts, lactic acid bacteria, sauerkraut, fermented food, gut microbiota, in vitro digestion

\section{INTRODUCTION}

Botanical fermented foods are microbially transformed plant products rich in health-promoting components. These non-dairy plant-based functional foods potentially manipulate the microbiotagut-metabolism axis (Mäkinen et al., 2016; Gille et al., 2018). As such, there has been an 
increase in research characterizing their extracted bioactive components (Değirmencioğlu et al., 2016; Septembre-Malaterre et al., 2018), isolated microbial strains, and their metabolites (Yu et al., 2013; London et al., 2014; Lara-Hidalgo et al., 2017; Angelescu et al., 2019; Romero-Luna et al., 2019, 2020). Few studies explore how being part of a whole fermented food matrix affects microbial viability during fermentation, storage, and gastrointestinal (GI) transit (Buriti et al., 2010; Fiorda et al., 2016; Valero-Cases et al., 2017; Yang et al., 2020). Even fewer investigate the effect of whole fermented food consumption on the gut microbiota (Lavefve et al., 2021). The human gut microbiota is an important dietary target, with its central regulatory role in immune function and energy metabolism (Gille et al., 2018). Botanical fermented foods are cheap, easily made, and consumed globally. This makes them excellent candidates for the dietary management of pro-inflammatory noncommunicable diseases, such as type 2 diabetes and metabolic syndrome.

Whole botanical fermented foods contain components that interact with gut bacteria, including microbes and their metabolites, prebiotic fibers, and other bioactive molecules (Marco et al., 2017). Their potential health effects are likely exerted by lactic acid bacteria (LAB) and yeasts, through biotransformation of inherent ingredients, production of microbial metabolites, or transient integration with gut bacteria (Gille et al., 2018). These microbes have been shown to display similar probiotic activity to dairy-based and human-related strains (La Anh, 2015; Tamang et al., 2016). Recent large-scale metagenomic studies suggest that fermented foods contain health-promoting LAB strains that are closely related to those in the gut microbiome and may well be an important source of commensal strains (Pasolli et al., 2020). In order to exert these potential benefits, LAB and yeasts must endure the rigors of the manufacturing process, storage, consumption, and GI transit (Forssten et al., 2011). Microbial survival in fermented foods is enhanced by integration with appropriate food delivery matrices, due to the presence of protective microbe-digestible sugars (Perrin et al., 2000), prebiotics, and complex microbial communities (Su et al., 2007; HernandezHernandez et al., 2012). To survive GI transit, microorganisms must possess intrinsic acid and bile tolerance (Food and Agriculture Organization of the United Nations and World Health Organisation, 2002; Derrien and van Hylckama Vlieg, 2015); food substrates act as acid buffering agents during digestion (Ranadheera et al., 2010). On reaching the gut, transient fermented food-associated microbes may integrate with gut commensals to produce immunomodulatory and anti-inflammatory metabolites (London et al., 2014; Lara-Hidalgo et al., 2017).

To the best of our knowledge, no studies have investigated the in vitro survival of $\mathrm{LAB}$ and yeasts and their impact on the gut microbiota when administered as a native part of botanical fermented foods. An accepted cost-effective and ethical way to determine microbial viability is the utilization of in vitro GI digestion studies to assess strain resistance to simulated gastric and enteric juices (Buriti et al., 2010; Gbassi et al., 2011). In vitro colonic fermentation with nextgeneration sequencing has proven useful for understanding the transformation and gut-level impacts of dietary compounds, including microbes, prebiotics, and polyphenols (Tsitko et al., 2019; Nissen et al., 2020). Two popular traditional botanical fermented foods, sugar-based tibicos and brine-based sauerkraut, contain potentially probiotic $\mathrm{LAB}$, including Lactiplantibacillus plantarum (formerly Lactobacillus plantarum), Levilactobacillus brevis (formerly Lactobacillus brevis), and Lactobacillus delbrueckii subsp. Lactis; (formerly Lactobacillus lactis), yeasts (most notably Saccharomyces spp.), and bioactive components (Marsh et al., 2013; Laureys and De Vuyst, 2014; Lavefve et al., 2021). In vitro tests of Saccharomyces cerevisiae strain C41 and Lacticaseibacillus paracasei CT12 (formerly Lactobacillus paracasei) isolated from tibicos showed similar probiotic activity to established comparable probiotic strains, with good resistance to gastric $\mathrm{pH}$, bile salts, and in vitro digestive fluids (Romero-Luna et al., 2017, 2019, 2020). Autochthonous LAB strains, including L. plantarum, from spontaneously fermented sauerkraut are known to have probiotic potential, due to their ability to resist a low acid environment, pancreatin, and bile salts (Yu et al., 2013). LAB strains extracted from sauerkraut have been shown to adhere to CaCo-2 cells and exert antibacterial activity against potential pathogens (Beganović et al., 2014). Leech et al. (2020) recently found that the microbial diversity of tibicos and sauerkraut far exceeded that of dairy-based ferments, as well as containing the largest numbers of potential health-promoting gene clusters. As such, tibicos, and sauerkraut were chosen for our study.

Growth and survival of LAB and yeasts is dependent on the microbial terroir during fermentation and storage: microbial species and strains, inherent food substrates, environmental $\mathrm{pH}$, and organic acid concentrations (Laureys et al., 2019). In the commercial sphere, functional spices, such as ginger, cayenne pepper, and turmeric, are often added to fermented products for sensory and purported health purposes; their effect on microbial proliferation and survival in fermented foods has not been adequately investigated. Similarly, low-salt sauerkraut is increasingly popular, but salt concentrations have been shown to affect microbial diversity and load in the finished product (Di Cagno et al., 2013; Septembre-Malaterre et al., 2018). Starter cultures are commonly used for sauerkraut fermentation and have also been shown to affect microbial growth (Pandey and Garg, 2015; Xiong et al., 2016). Assessing these relationships with physicochemical and in vitro systems allows us to develop botanical fermented foods with maximal beneficial impact on intestinal bacteria and thus human health.

In this study, we investigate the effects of storage length, fermentation processes (including salt concentration and use of starter cultures), and functional spices cayenne, ginger, and turmeric on the survival of $\mathrm{LAB}$ and yeasts in whole naturally fermented tibicos and sauerkraut, and the subsequent impact on gut bacterial relative abundance in a static in vitro digestion and porcine colonic fermentation model. Our findings provide valuable insights into microbial viability in botanical fermented foods from production to consumption, as well as contributing to a useful base for further human studies. 


\section{MATERIALS AND METHODS}

\section{Tibicos and Sauerkraut Production}

Cabbages were purchased from a local retailer and sliced finely before placing into fermentation vessels. Fermentation was carried out anaerobically at $22-25^{\circ} \mathrm{C}$ for 7 days, then stored at $4^{\circ} \mathrm{C}$ for sauerkraut ripening. Three sauerkraut treatments were produced in triplicate: (1) spontaneous fermentation with $0.6 \% \mathrm{NaCl}$ and cabbage; (2) spontaneous fermentation with $1.5 \% \mathrm{NaCl}$ and cabbage; and (3) inoculated fermentation with $0.091 \%(w / w)$ starter cultures (labeled as L. plantarum, Leuconostoc mesenteroides, and Pediococcus acidilactici; Caldwell, Canada), $0.6 \% \mathrm{NaCl}$ and cabbage. The sauerkraut treatments were fermented at $20-25^{\circ} \mathrm{C}$ for 7 days, then stored at $4^{\circ} \mathrm{C}$ for a further 88 days. During fermentation and storage, brine $(10 \mathrm{ml})$ and cabbage $(10 \mathrm{~g})$ samples were withdrawn aseptically on days $0,1,3,7,13,19,34,47$, and 95 for $\mathrm{pH}$ measurements and microbiological tests. Samples were homogenized and extracts collected, then stored at $-20^{\circ} \mathrm{C}$ for further chemical composition determination.

The primary fermentation of tibicos was performed to ensure that the grains were active and fresh for the subsequent fermentation. Tibicos grains were sourced from a local producer. For every $60 \mathrm{~g}$ of wet-weight of tibicos grains, $1 \mathrm{~L}$ of $10 \%$ $(w / v)$ sterilized sucrose solution was supplemented with half an organic dried fig. Tibicos mixtures were incubated at $20-25^{\circ} \mathrm{C}$ for $72 \mathrm{~h}$, after which the tibicos were separated from the liquor and recultivated in new sucrose solution under the same conditions. Tibicos from the primary fermentations were evenly divided into sterilized bottles. Four tibicos treatments were prepared in triplicate: (1) plain tibicos without botanic flavoring powder; (2) ginger tibicos with $0.5 \%(w / v)$ organic ginger powder (Zingiber officinale); (3) cayenne tibicos with $0.125 \%(w / v)$ organic cayenne powder (Capsicum frutescens); and (4) turmeric tibicos with $0.25 \%(w / v)$ organic turmeric powder (Curcuma longa). All botanic flavoring powders were purchased from a local retailer. After the $72-\mathrm{h}$ primary fermentation with tibicos grains, flavoring powders were added and the four treatments were anaerobically incubated at $20-25^{\circ} \mathrm{C}$ for $48 \mathrm{~h}$, then stored at $4^{\circ} \mathrm{C}$ for a further 91 days. Samples were aseptically extracted $(1 \mathrm{ml})$ every $24 \mathrm{~h}$ of primary fermentation and on storage days 12, 19, 33, 47, and 96 for $\mathrm{pH}$ measurements and microbiological tests. Length of storage was based on the commercial shelf lives of these products.

\section{Microbial Enumeration}

Viable microbial counts were determined using the methods of De Angelis et al. (2015) and Fischer et al. (2014) with slight modifications. To enumerate LAB, yeasts, and molds, serial dilutions of samples were plated onto modified de Man, Rogosa, and Sharpe (MRS) agar (with $4 \mathrm{mg} / \mathrm{L}$ cycloheximide) and yeast extract peptone dextrose medium (YPD; with $10 \mathrm{mg} / \mathrm{L}$ chloramphenicol and $100 \mathrm{mg} / \mathrm{L}$ ampicillin). For some samples, Lactobacillus anaerobic MRS agar with vancomycin and bromocresol green (LAMVAB) agar was also used for LAB enumeration. All types of agar plates were incubated in a constant temperature incubator at $28^{\circ} \mathrm{C}$ for 3 days.

\section{Chemical Analysis}

The $\mathrm{pH}$ was determined with a Hanna HI $5221 \mathrm{pH}$ meter (Hanna Instrument, Melbourne, Australia). Corresponding enzymatic assay kits from Megazyme, Bray, Ireland ${ }^{1}$ were used to measure lactate (K-LATE) and acetate (K-ACET); sucrose, glucose and fructose (K-SUFRG); ethanol (K-ETOH), mannitol (K-MANOL), and glycerol (K-GCROL); and ascorbic acid content (K-ASCO). All reagents came from the enzymatic kit and determination processes were conducted based on the manufacturer's instructions with some modifications for the determination of sugars.

\section{Simulated Upper Gastrointestinal Digestion and Colonic Fermentation}

The simulated upper gastrointestinal digestion and colonic fermentation tests were conducted according to a combination of the consensus harmonized static in vitro digestion model (Minekus et al., 2014) and the protocol by Sirisena et al. (2018) with slight modifications (below). We used feces from healthy pigs for colonic fermentation. Samples of both sauerkraut and tibicos were taken on day 34 for administration to the in vitro digestive system. This time point ensured that there was a high microbial load (around 7-8 log cfu/ml) and considered the typical length of storage in a commercial setting.

\section{Digestive Fluids and Fecal Slurry}

Simulated saliva fluid (SSF), simulated gastric fluid (SGF), simulated intestinal fluid (SIF), enzyme (amylase, porcine pepsin, pancreatin, and bile) solution, $0.1 \mathrm{M}$ phosphate buffer, basal media, and fecal slurry were prepared according to the methods prescribed by Sirisena et al. (2018). All digestive fluids, enzyme solutions, and fecal slurries were utilized within $1 \mathrm{~h}$ of preparation.

\section{In vitro Digestion Simulation}

\section{Oral Digestion}

The oral stock solution consisted of $1.75 \mathrm{ml}$ of SSF solution, $0.25 \mathrm{ml}$ fresh salivary $\alpha$-amylase solution of $1,500 \mathrm{U} / \mathrm{ml}$ (dilute the original solution with SSF solution), $12.5 \mu \mathrm{l}$ of $0.3 \mathrm{~mol} / \mathrm{l}$ $\mathrm{CaCl}_{2}\left(\mathrm{H}_{2} \mathrm{O}\right)_{2}$, and $487.5 \mu \mathrm{l}$ of water. To this mixture, $1 \mathrm{ml}$ of sauerkraut brine and $1.5 \mathrm{~g}$ finely minced sauerkraut, or $2.5 \mathrm{ml}$ of tibicos was added to achieve the final ratio of sample: stock solution $=1: 1$. All tubes were tightly sealed, then mixed by a pre-warmed $37^{\circ} \mathrm{C}$ shaking incubator with a constant parameter of $200 \mathrm{rpm}$ for $2 \mathrm{~min}$. An oral bolus was obtained.

\section{Gastric Digestion}

The $5 \mathrm{ml}$ oral bolus was mixed with $3.75 \mathrm{ml}$ of SGF solution, $0.8 \mathrm{ml}$ fresh porcine pepsin stock solution of $25,000 \mathrm{U} / \mathrm{ml}$ (dissolved in SGF solution), $2.5 \mu \mathrm{l}$ of $0.3 \mathrm{~mol} / 1 \mathrm{CaCl}_{2}\left(\mathrm{H}_{2} \mathrm{O}\right)_{2}$, and $387.5 \mu \mathrm{l}$ of Milli-Q water. The final sample-to-stock ratio

${ }^{1}$ www.megazyme.com 
was $1: 1$. The mixture $\mathrm{pH}$ was adjusted to 3.0 via the addition of required $\mathrm{HCl}$. All tubes then underwent shaking for $2 \mathrm{~h}$ at $37^{\circ} \mathrm{C}$. Gastric chyme was obtained.

\section{Small Intestinal Digestion}

The gastric chyme was mixed with $5.5 \mathrm{ml}$ SIF solution, $2.5 \mathrm{ml}$ of fresh pancreatin solution of $800 \mathrm{U} / \mathrm{ml}$ (pre-warmed to $37^{\circ} \mathrm{C}$ and well-shaken), $1.25 \mathrm{ml}$ fresh fed state bile $(49 \mathrm{mg} / \mathrm{ml}), 20 \mu \mathrm{l}$ of $0.3 \mathrm{~mol} / \mathrm{L} \mathrm{CaCl}{ }_{2}\left(\mathrm{H}_{2} \mathrm{O}\right)_{2}$, and $655 \mu \mathrm{l}$ (sauerkraut) or $1.152 \mathrm{ml}$ (tibicos) of Milli-Q water. The $\mathrm{pH}$ of the mixture was adjusted to 7.0 via addition of $1 \mathrm{~mol} / \mathrm{l}$ of $\mathrm{NaOH}$, before $2 \mathrm{~h}$ shaking incubation at $37^{\circ} \mathrm{C}$. For sauerkraut samples, the small intestinal digesta was centrifuged at $5,000 \mathrm{rpm}$ for $15 \mathrm{~min}$. $1 \mathrm{ml}$ of sauerkraut small intestinal digesta was collected for microbiological determination. As the tibicos sample was a clear liquid containing minimal solids, $1 \mathrm{ml}$ of the small intestinal digesta was directly sampled and stored, rather than being centrifuged to acquire precipitate fractions. Precipitate fractions were separated and stored at $4^{\circ} \mathrm{C}$ for colonic fermentation.

\section{Microbial Enumeration}

Triplicate samples of all treatments prior to and following gastric and small intestinal digestion were serially diluted. One hundred microliter of each dilution was spread-plated on MRS, YPD, and LAMVAB agar for aerobic and anaerobic microbial enumeration. Triplicate blanks - fecal samples without small intestinal digesta - were also diluted to enumerate the original existing $\mathrm{LAB}$ and yeast in porcine feces. The plates were then incubated.

\section{Colonic Fermentation}

Here, $1 \mathrm{ml}$ tibicos small intestinal digesta or $1 \mathrm{ml}$ sauerkraut precipitate fraction was mixed with $5 \mathrm{ml}$ each of pre-warmed basal medium and fecal slurry (Sirisena et al., 2018). All tubes were flushed with nitrogen gas for several seconds to remove air. This was followed by a $24 \mathrm{~h}$ fermentation at $37^{\circ} \mathrm{C}$ in the anaerobic chamber of a shaking incubator. An aliquot of $1 \mathrm{ml}$ from each resulting liquid solution was taken for microbiological tests, while the remainder was frozen at $-20^{\circ} \mathrm{C}$ for DNA extraction and sequencing.

\section{DNA Extraction and Sequencing}

Genomic DNA was extracted from the duplicated colonic fermentation samples, using PowerSoil ${ }^{\mathrm{TM}}$ DNA Isolation kits (QIAgen, CA, United States), as per the kit protocol. A blank (fecal slurry with digestive fluids and no fermented food) and a control (colonic digesta) were included. DNA extracts were submitted to the Australian Genome Research Facility (AGRF) for amplification and sequencing. To assess the bacterial communities, 16S rRNA gene V3-V4 region was amplified using the universal primer pairs 341F/806R (Yu et al., 2005), followed by $300 \mathrm{bp}$ paired-end sequencing on an Illumina MiSeq (San Diego, CA, United States). Raw sequences were processed using QIIME v1.9.2 (Caporaso et al., 2010). Operational taxonomic units (OTUs) were assigned using UCLUST openreference OTU-picking workflow with a threshold of $97 \%$ pairwise identity (Edgar, 2010). Taxonomy was assigned to OTUs in QIIME using the Ribosomal Database Project (RDP) classifier (Wang et al., 2007) against the GreenGenes bacterial 16S rRNA database (v13.8; DeSantis et al., 2006).

\section{Data and Statistical Analysis}

All experiments were performed in triplicate, with results reported as the mean \pm standard deviation. Differences among samples were assessed using one-way ANOVA and Fisher pairwise comparisons at a significance level of 0.05 using Minitab18.

\section{RESULTS}

Our study evaluated microbial viability of LAB and yeasts in sauerkraut and tibicos during fermentation and storage, using a plate colony-counting method. $\mathrm{pH}$ value and chemical composition changes were tested using appropriate methods. Static in vitro digestion tests were performed to determine microbial survival in a simulated gastric and small intestinal model. Finally, colonic fermentation was used to determine the effect of LAB and yeast on porcine gut bacteria.

\section{pH and Chemical Composition}

For all tibicos and sauerkraut treatments, we measured organic acid (lactic acid, acetic acid, and ascorbic acid), alcohol (ethanol, glycerol, and mannitol), and sugar (fructose, glucose, and sucrose) content, and $\mathrm{pH}$, during fermentation and storage (Supplementary Material). During early fermentation, the $\mathrm{pH}$ of both sauerkraut and tibicos dropped below 3.5. In tibicos, lactic and acetic acid levels increased, as did ethanol, glycerol, and mannitol levels, while all carbohydrate levels fell. In sauerkraut, lactic and acetic acid levels increased, as did ethanol and mannitol levels, while ascorbic acid levels dropped. During tibicos storage, $\mathrm{pH}$ trended downward, falling to around 3. Lactic and acetic acid levels dropped slightly, then gradually increased and plateaued at day 50, with only ginger tibicos experiencing a continuing upward trend. Ethanol, glycerol and mannitol levels dipped then increased and plateaued at day 50. During sauerkraut storage, $\mathrm{pH}$ remained stable. Lactic and acetic levels increased, except for the inoculated sauerkraut which showed a precipitous drop in acetic acid from day 34 onward. Ascorbic acid levels of all sauerkraut continued to decline. Ethanol levels increased, except in inoculated sauerkraut where it plateaued; mannitol levels in all sauerkraut plateaued. Significant differences between the treatments were not found $(p>0.05)$.

\section{Microbial Growth and Survival During Fermentation and Storage}

Our study investigated LAB and yeast survival during fermentation and storage. During the 7-day fermentation and a further 88 days of storage, samples were taken, diluted, and plated for microbial enumeration (Figure 1).

Sauerkraut made with different salt concentrations did not show any significant difference in LAB counts $(p>0.05)$. The inoculation of starter culture increased initial LAB counts by 


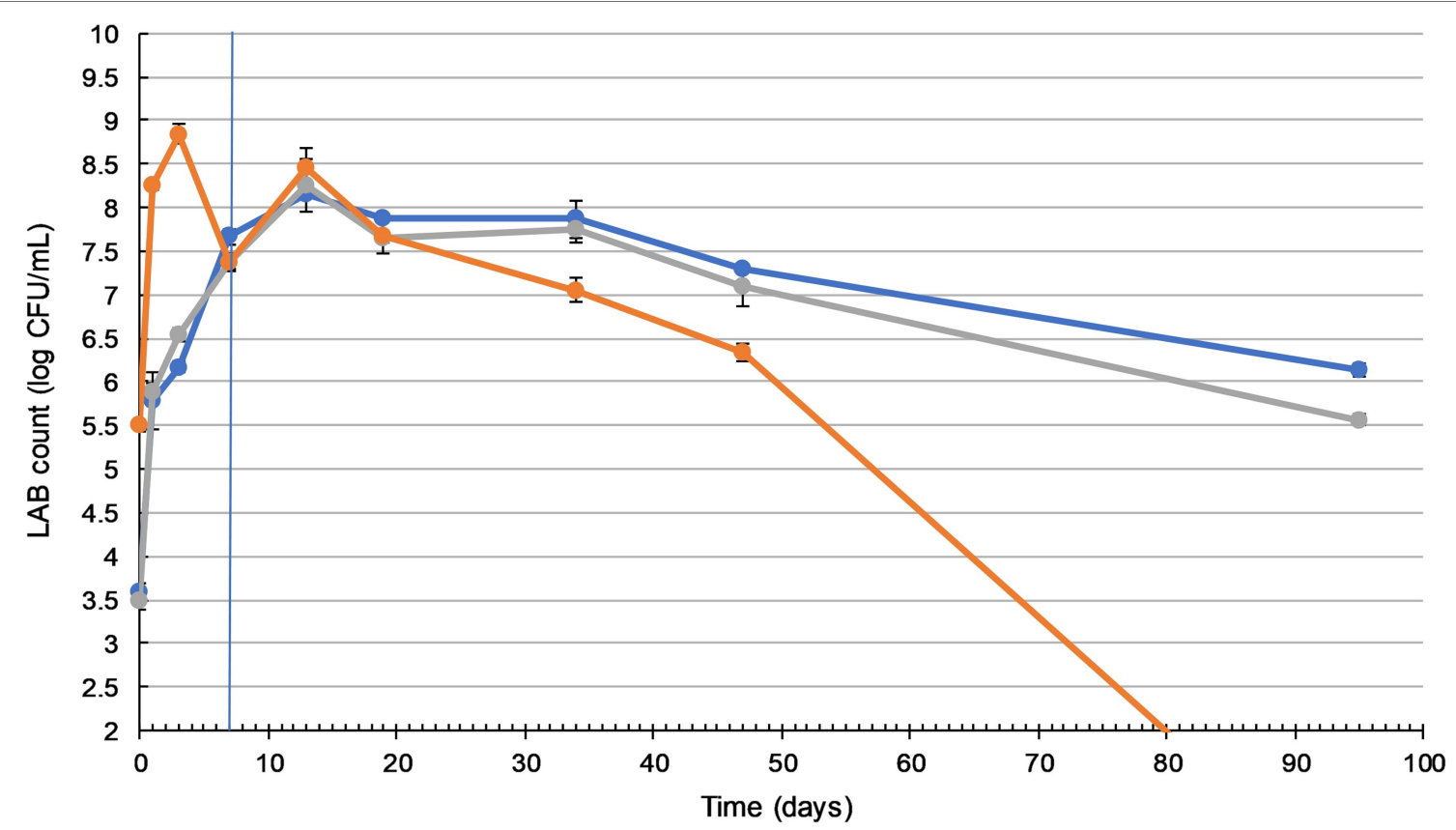

FIGURE 1 | Lactic acid bacteria (LAB) counts in sauerkraut remain sufficiently high during storage. Changes in $L A B$ count during fermentation (20-25 ${ }^{\circ} \mathrm{C}$ for 7 days) and storage $\left(4^{\circ} \mathrm{C}\right.$ for a further 88 days) of three sauerkraut treatments. ( - ) spontaneous fermentation with $0.6 \%$ salt; $(-\infty)$ spontaneous fermentation with $1.5 \%$ salt; (-) inoculated fermentation with $0.91 \%(w / w)$ starter cultures and 0.6\% salt. Sampled on days 0, 1, 3, and 7 (during fermentation), and on days $13,19,34$, 47 , and 95 . The timeline of fermentation and storage is separated by the vertical blue line. The results were expressed as mean standard deviation ( $n=3)$ for each treatment group.

more than $2 \log$ units, from 3.5 to $5.5 \log \mathrm{cfu} / \mathrm{ml}$. Inoculated and spontaneously fermented sauerkraut then followed a similar LAB count trend, with a significant gradual increase $(p<0.05)$ to a peak of around $8 \mathrm{log} \mathrm{cfu} / \mathrm{ml}$ on day three (inoculated), during fermentation, and day 13 (spontaneous), during storage. Inoculated sauerkraut $\mathrm{LAB}$ counts dropped to undetectable levels by the end of storage, while spontaneously fermented sauerkraut LAB counts remained stable at around 6-7 log cfu/ $\mathrm{ml}$ from day 47 to 95 .

Yeast enumeration was also carried out (Supplementary Material), with a peak of around $5.6 \mathrm{log} \mathrm{cfu} / \mathrm{ml}$ and no significant difference between the treatments $(p>0.05)$. By the 3rd day of fermentation, no yeast or other molds were detected in any of the sauerkraut samples.

The LAB count in all tibicos treatments significantly increased during fermentation $(p<0.05)$, with slight fluctuations on addition of functional spices (Figure 2A). There was no significant difference between the treatments after 5 days of fermentation $(p>0.05)$. During storage, the LAB count of all treatments increased, reaching their peak of around $7 \log \mathrm{cfu} / \mathrm{ml}$ on day 19 for cayenne, day 35 for plain and day 47 for ginger and turmeric tibicos. The LAB count of ginger tibicos remained stable and was significantly higher at $7 \log \mathrm{cfu} / \mathrm{ml}$ than the other three treatments on day $96(p<0.05)$. The LAB count of the other three treatments gradually decreased to around 6-6.5 $\mathrm{log} \mathrm{cfu} / \mathrm{ml}$. Overall, LAB content in tibicos increased 60-fold over 96 days (Figure 2A).

Yeast counts increased slightly during the initial fermentation, peaking at around $6 \mathrm{log} \mathrm{cfu} / \mathrm{ml}$ (Figure 2B). During secondary fermentation, the yeast counts of the ginger and cayenne tibicos did not differ from each other but were significantly higher than the plain and turmeric tibicos $(p<0.05)$. During early storage, the yeast counts of all four tibicos treatments experienced a plateau, followed by a significant surge to their highest points of around $7 \log \mathrm{cfu} / \mathrm{ml}$, followed by a slow decline over time. In the last month of storage, yeast counts of plain, cayenne, and turmeric tibicos dropped significantly to around $6 \log \mathrm{cfu} /$ $\mathrm{ml}$. Ginger tibicos yeast counts remained stable at approximately $7 \mathrm{log} \mathrm{cfu} / \mathrm{ml}$. On day 96, ginger tibicos had significantly higher yeast counts than the other three treatments $(p<0.05$; Figure $2 B$ ).

\section{Survival of LAB and Yeasts Under Simulated GI Conditions}

Microbial enumeration showed that LAB in sauerkraut and tibicos were able to survive fermentation and periods of storage in sufficient probiotic numbers. Our study then investigated if these microbes could tolerate low $\mathrm{pH}$, bile salts, pepsin, and pancreatin in a simulated gastric and small intestinal model.

Prior to in vitro digestion, there were no significant differences in LAB counts between sauerkraut treatments (Figure 3), all of which were around $8 \log \mathrm{cfu} / \mathrm{ml}$. The LAB count of the unfermented cabbage and salt mixture was around $3 \log \mathrm{cfu} / \mathrm{ml}$. After the small intestinal phase, LAB counts of all the sauerkraut treatments were significantly lower than prior to GI simulation $(p<0.05)$, dropping to around $6 \log \mathrm{cfu} / \mathrm{ml}$. The LAB count of the unfermented cabbage treatment increased, but not significantly. There were no 

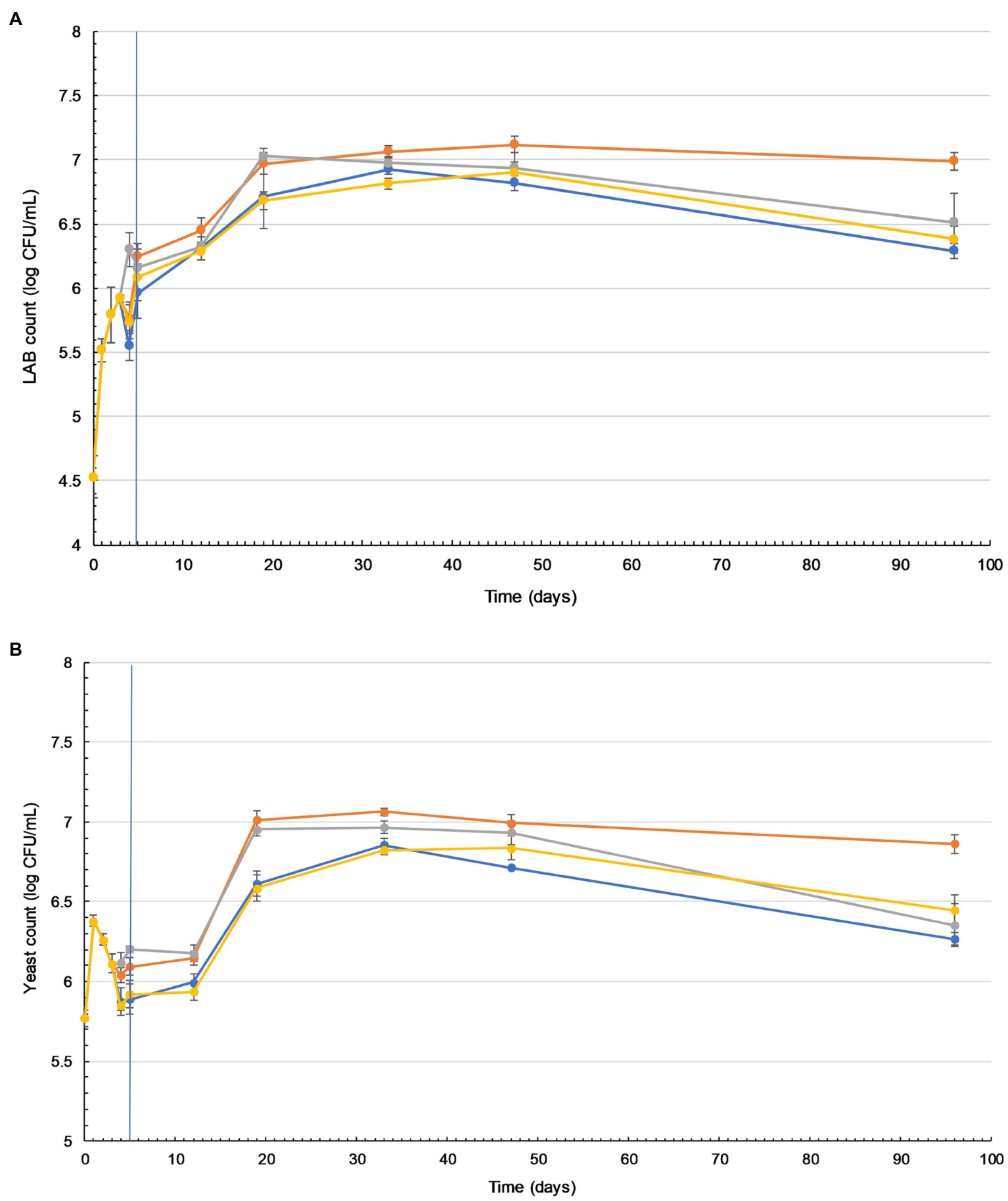

FIGURE 2 | Lactic acid bacteria (LAB) and yeast counts in tibicos increase during storage and are significantly higher when ginger is added. Changes in viable LAB (A) and yeast (B) counts in four tibicos treatments during fermentation (20-25 $\mathrm{C}$ for 3 days with tibicos; and with three different additives added on day 3 for 2 days of secondary fermentation without tibicos and storage at $4^{\circ} \mathrm{C}$ for a further 86 days). (-) Plain tibicos; ( --$)$ tibicos with $0.5 \%$ ( $\left.w / v\right)$ organic ginger powder; $(-)$ tibicos with $0.125 \%(w / v)$ organic cayenne powder; $(--)$ tibicos with $0.25 \%(w / v)$ organic turmeric powder. Sampled daily during fermentation and on days $12,19,33,47$, and 96 . The timeline of fermentation with the tibicos grains and separation to enter storage is indicated by the vertical blue line. The results were expressed as mean standard deviation $(n=3)$ for each treatment group.

significant differences between the sauerkraut treatments, though their LAB counts were all significantly higher than the unfermented cabbage $(p<0.05)$. The survival rate of LAB was not significantly different between sauerkraut treatments at around 72\% (Table 1), but the unfermented cabbage treatment had a significantly higher survival rate of LAB than the sauerkraut treatments $(p<0.05)$. 


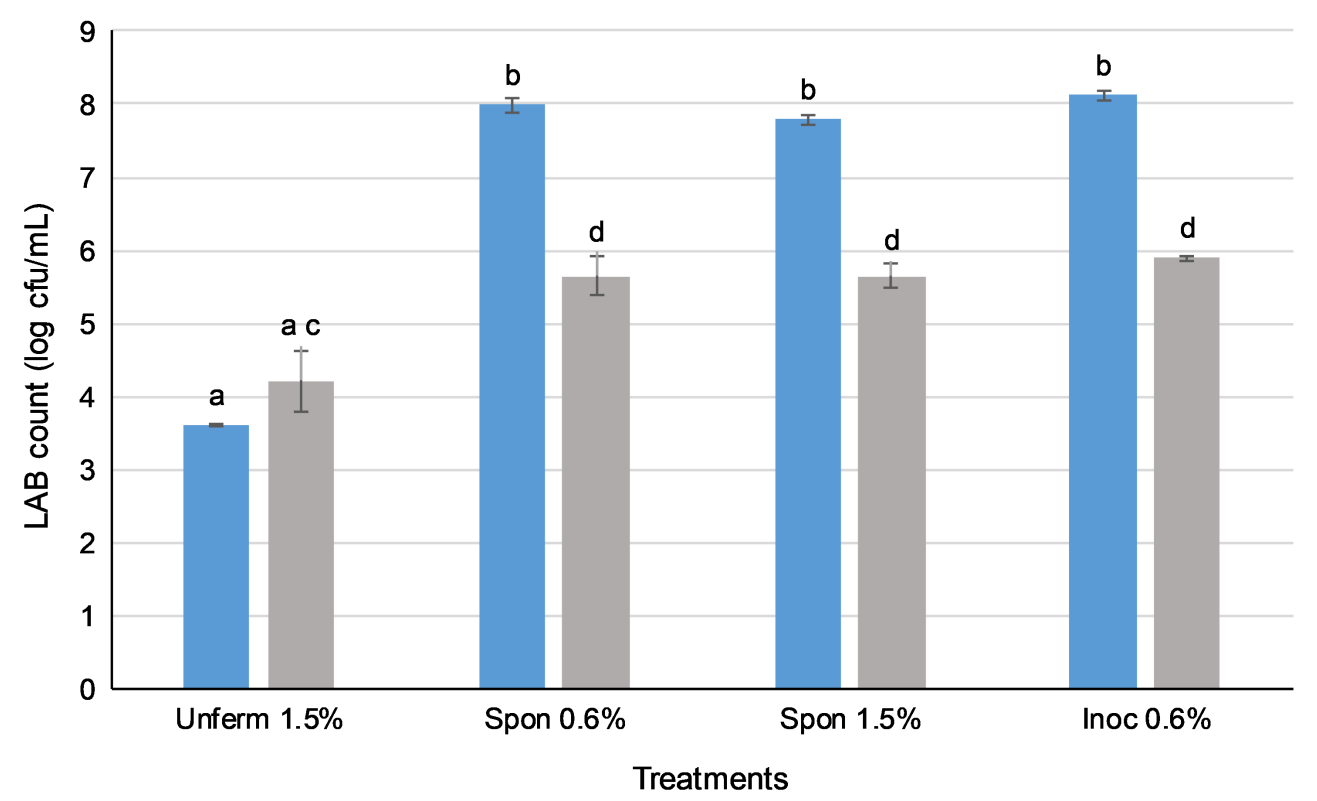

FIGURE 3 | Lactic acid bacteria in sauerkraut survive simulated oral, gastric, and small intestinal conditions. $\square$ ) Total $L A B$ plate counts before in vitro digestion; ( $\square$ ) total LAB plate counts after simulated Gl conditions. The results were expressed as mean standard deviation $(n=3)$ for each treatment group. Means with the same letter are not significantly different $(p<0.05)$.

TABLE 1 | Salt concentration and inoculation do not affect lactic acid bacteria $(\mathrm{LB})$ survival rate during in vitro digestion.

\begin{tabular}{lc}
\hline Treatments & LAB survival rate \\
\hline Unfermented cabbage $+1.5 \%$ salt & $117.1 \pm 0.12 .3^{\mathrm{a}}$ \\
Spontaneously fermented sauerkraut $+0.6 \%$ salt & $71.7 \pm 2.3^{\mathrm{b}}$ \\
Spontaneously fermented sauerkraut $+1.5 \%$ salt & $72.5 \pm 1.7^{\mathrm{b}}$ \\
Inoculated sauerkraut $+0.6 \%$ salt & $72.7 \pm 0.5^{\mathrm{b}}$
\end{tabular}

Percentage survival of sauerkraut-associated $L A B$ after simulated gastric and small intestinal digestion. Means within a column with different superscripts $(a, b)$ are significantly different $(p<0.05)$. The results expressed as mean $(n=3) \pm S D$ (standard deviation).

In all four tibicos treatments, both $\mathrm{LAB}$ and yeast counts fell significantly after the small intestinal phase ( $p<0.05$; Figure 4). $\mathrm{LAB}$ counts experienced an approximate $2 \log \operatorname{loss}$ to $5 \log$ $\mathrm{cfu} / \mathrm{ml}$, while yeast counts dropped by $1 \log$ to $6 \log \mathrm{cfu} / \mathrm{ml}$. Cayenne tibicos had a significantly higher LAB count compared to plain and turmeric tibicos $(p<0.05)$, and ginger tibicos had a significantly higher LAB count than turmeric tibicos $(p<0.05)$. Ginger tibicos had significantly higher yeast count than both plain and turmeric tibicos, while cayenne tibicos had significantly higher yeast counts than turmeric tibicos. The addition of cayenne powder to tibicos significantly improved the survival rate of LAB during simulated gastric and small intestinal digestion $(p<0.05)$, compared to ginger and turmeric. Ginger tibicos had a significantly higher rate of LAB survival than turmeric tibicos $(p<0.05)$, but neither treatment had significantly higher LAB survival rates than plain tibicos $(p<0.05)$. Yeast survival rates in ginger and turmeric tibicos were significantly lower than plain and cayenne tibicos $(p<0.05)$.

\section{Impact of Digested LAB and Yeasts on Gut Microbiota}

$\mathrm{LAB}$ and yeasts from the fermented foods were able to survive simulated digestion in high numbers, but we wanted to know if these microbes could affect the complex microbial community of the large intestine. To observe these effects, after in vitro gastric and small intestinal digestion, all tibicos and sauerkraut samples then underwent 24-h colonic fermentation using porcine feces. Bacterial communities were profiled by extracting DNA from all samples and performing $16 \mathrm{~S}$ rRNA amplicon sequence analysis. A total of 861,164 16S rRNA high-quality sequences were generated from all the samples, which were clustered into 1,891 bacterial OTUs with a threshold of $97 \%$ pairwise identity.

\section{Microbial Enumeration}

The fecal slurry contained $11 \log \mathrm{cfu} / \mathrm{ml} \mathrm{LAB}$ and $10.5 \log \mathrm{cfu} /$ $\mathrm{ml}$ yeast. All tibicos and sauerkraut small intestinal digesta were mixed with the fecal slurry before colonic fermentation. After colonic fermentation, the control contained $8 \mathrm{log} \mathrm{cfu} / \mathrm{ml} \mathrm{LAB}$ and $5.6 \log$ $\mathrm{cfu} / \mathrm{ml}$ yeast. Tibicos samples contained 7-8 $\log \mathrm{cfu} / \mathrm{ml} \mathrm{LAB}$ and $6 \mathrm{log} \mathrm{cfu} / \mathrm{ml}$ yeast; sauerkraut samples had $8 \mathrm{log} \mathrm{cfu} / \mathrm{ml} \mathrm{LAB}$ and 5-6 log cfu/ml yeast remaining (Supplementary Material).

\section{Taxonomic Composition of Blank and Control Samples}

In the blank fecal samples, Bacteroidetes, Firmicutes, and Spirochetes made up $>97 \%$ of the phyla detected (Figures 5, 6). The relative abundance of the phylum Firmicutes was higher than Bacteroidetes (Figures 5A, 6A). At the family level (Figures 5B, 6B), Ruminococcaceae had the highest relative 


\section{A}

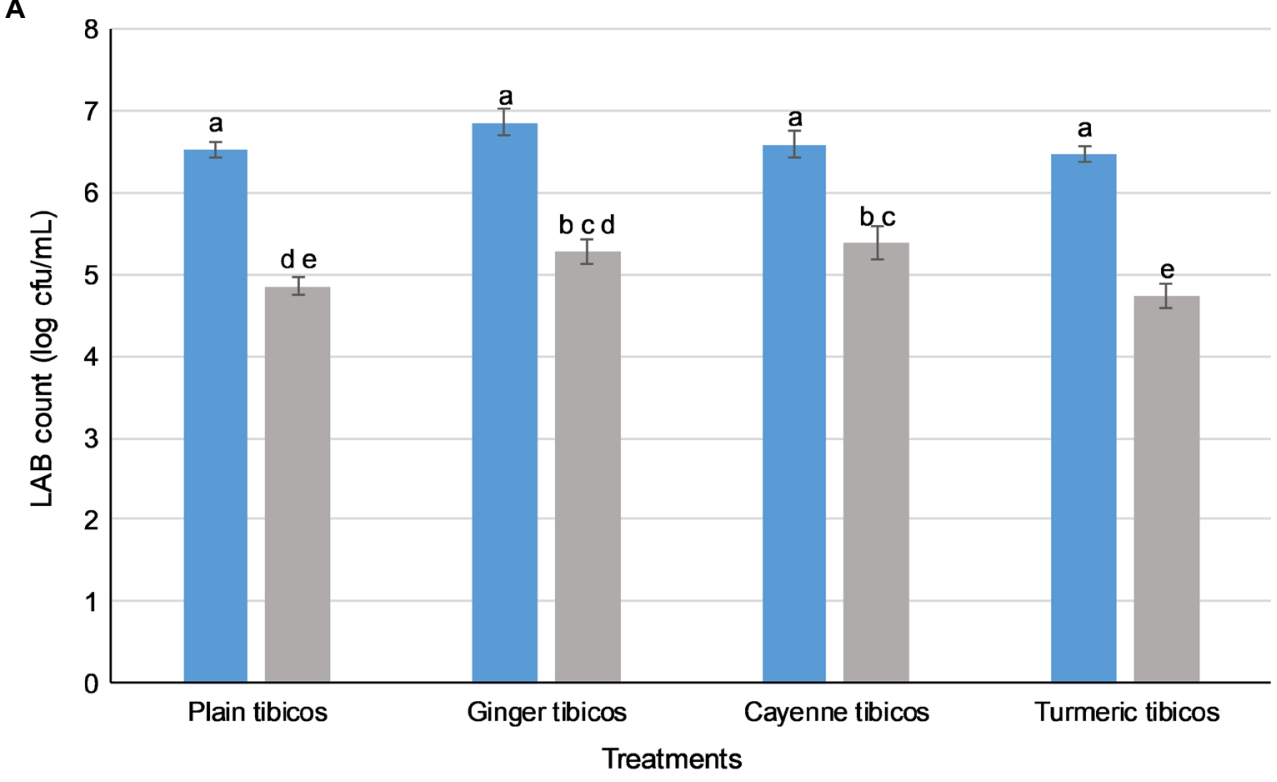

B

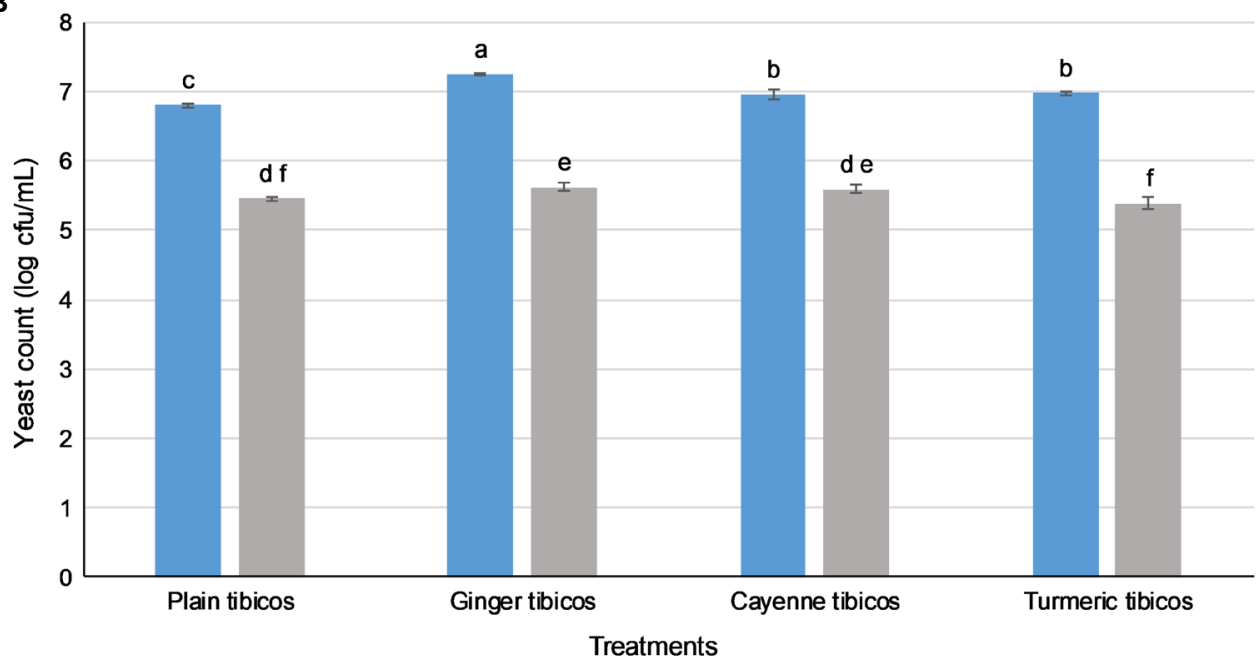

FIGURE 4 | Lactic acid bacteria ( $L A B)$ and yeasts in tibicos survive simulated oral, gastric, and small intestinal conditions. LAB (A) and yeast (B) counts. ( $\square$ ) LAB or yeast counts before in vitro digestion; ( each treatment group. Means with the same letter are not significantly different $(p<0.05)$.

abundance at around 14\%, with Muribaculaceae, Prevotellaceae, Spirochaetaceae, and Clostridiaceae at between 8 and 10\% each. At the genus level, Prevotella dominated at $11 \%$, with Streptococcus relative abundance (Figures 5C, 6C) at 9\%. Lactobacillus relative abundance was at $0.4 \%$ and Bifidobacterium at $0.07 \%$, while Enterobacter had 0\% relative abundance.

After colonic fermentation, Firmicutes, Proteobacteria, and Fusobacteria comprised $>91 \%$ of the phyla detected in the control, with relative abundance of Bacteroidetes dropping from 40 to $5 \%$ (Figures 5A, 6A). The relative abundance of Firmicutes was much higher than Bacteroidetes after simulated colonic digestion. At the family level, in comparison with the blank, Enterobacteriaceae (0.7\% vs. 34\%) (Figures 5B, 6B), Fusobacteriaceae (0.01\% vs. $14 \%$ ), and Lachnospiraceae (6 vs. 11\%) dominated. Lactobacillaceae increased tenfold from 0.4 to $5 \%$. At the genus level (Figures 5C, 6C), compared to the blank, the dominant genera were Escherichia (0.7-33\%), Fusobacterium (0.01-14\%), and Sharpea $(0.02-8 \%)$. Lactobacillus relative abundance increased from 0.4 to $5 \%$. The relative abundance of several families decreased: Clostridium (3\% to 0.5), Streptococcus (9-4\%), Prevotella (11-0.6\%), and Treponema (7-0.08\%).

\section{Taxonomic Composition of Sauerkraut and Tibicos Colonic Digesta}

Relative abundance across all sauerkraut treatments was at $<1 \%$ on phylum and family levels; at genus level, relative abundance was at $<0.1 \%$ across all treatments (Figure 5). After colonic digestion, Firmicutes, Proteobacteria, and Fusobacteria comprised 


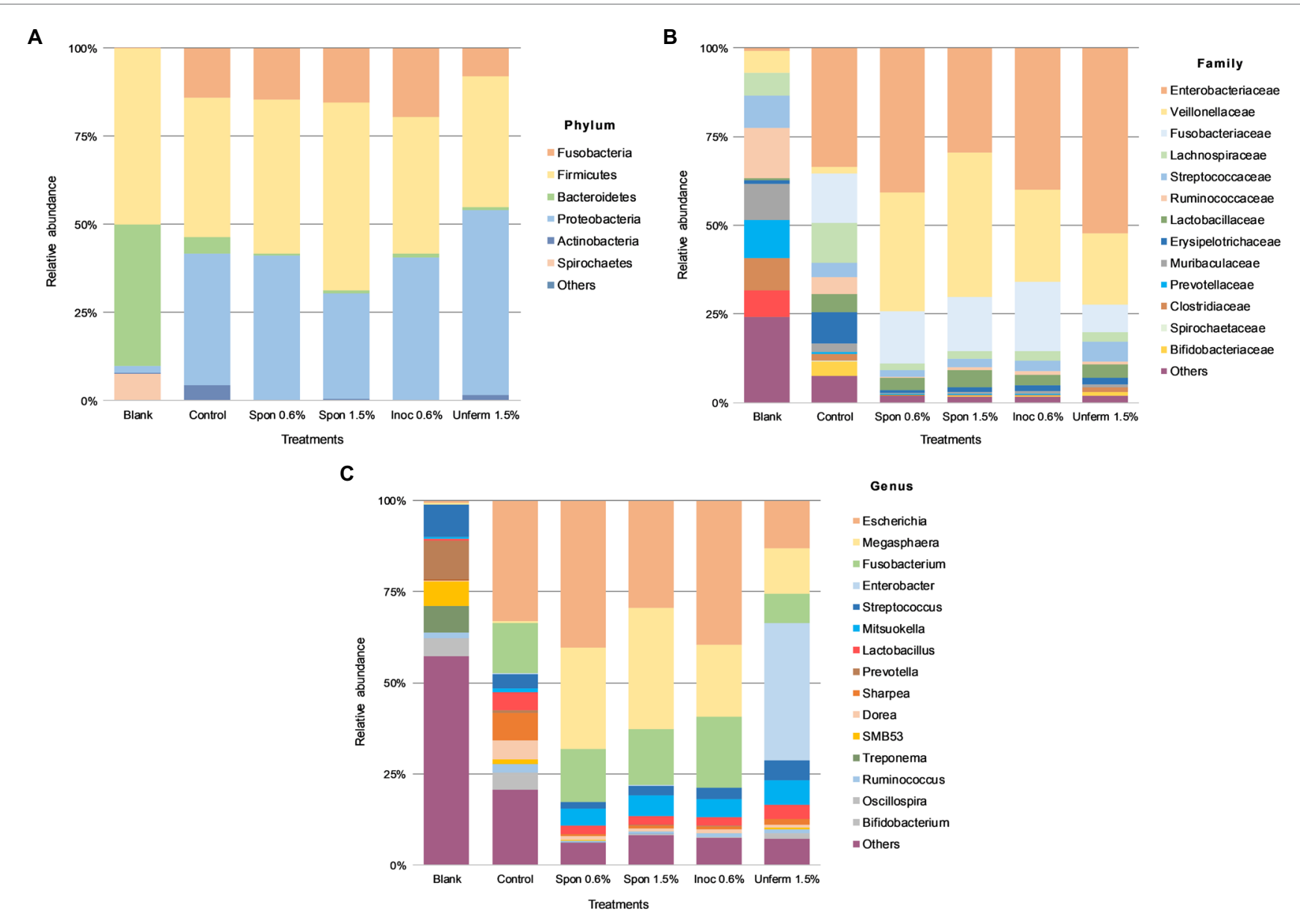

FIGURE 5 | Colonic fermentation of digested sauerkraut affects the final gut bacterial profile. Microbial taxa are characterized to the phylum (A); family (B); genus (C) levels. Dominant bacterial taxa are given with greater than $1.0 \%$ relative abundance. Blank: fecal slurry with digestive fluids. Control: fecal slurry with digestive fluids post-colonic fermentation. Spon $0.5 \%$ : spontaneously fermented sauerkraut with $0.6 \%$ salt; Spon $1.5 \%$ : spontaneously fermented sauerkraut with $1.5 \%$ salt; Inoc $0.6 \%$ : inoculated sauerkraut with $0.6 \%$ salt; Unferm $1.5 \%$ : unfermented cabbage with $1.5 \%$ salt.

$>98 \%$ of phyla detected in the unfermented cabbage and all sauerkraut treatments. These phyla also dominated in the control sample. Compared to the control at $5 \%$, Bacteroidetes relative abundance dropped to between 0.3 and $1 \%$; Firmicutes relative abundance in the control was at $40 \%$ compared to the treatments which were between 37 and 53\%. At the family level (Figure 5B), like the control, Enterobacteriaceae had the highest relative abundance in all sauerkraut treatments (between 40 and 52\%); Veillonellaceae expanded from $1.8 \%$ in the control, to between 20 and $34 \%$ in sauerkraut samples. The exception was spontaneously fermented sauerkraut with $1.5 \%$ salt, where Veillonellaceae was at $40 \%$ relative abundance and Enterobacteriaceae at $30 \%$. This also differed from the control where Enterobacteriaceae, Fusobacteriaceae and Lachnospiraceae dominated. Like the control, Lactobacillaceae relative abundance increased compared to the blank. At the genus level (Figure 5C), like the control, Escherichia was most abundant. Compared to the control (0.4\%), Megasphaera relative abundance increased around 85 -fold in the spontaneously fermented sauerkraut (28 and $33 \%$ ) and to $20 \%$ in the inoculated sauerkraut and $13 \%$ in the unfermented cabbage. Fusobacterium was also relatively dominant at $15-20 \%$ in the sauerkraut samples and $8 \%$ in the unfermented cabbage. In the unfermented cabbage, Enterobacter dominated at $38 \%$, in contrast to the control and sauerkraut treatments. Compared to the control at 5\%, sauerkraut Lactobacillus relative abundance was halved, while Bifidobacterium relative abundance dropped from $4 \%$ to around $0.2 \%$. In the unfermented cabbage treatment, there was less of a reduction compared to control, with $4 \%$ Lactobacillus and $1 \%$ Bifidobacterium relative abundance.

Relative abundance across all tibicos treatments was at $<1 \%$ on phylum, genus, and family levels (Figure 6). Like the control colonic digesta, Firmicutes, Fusobacteria, and Proteobacteria comprised $>97 \%$ of phyla detected in the tibicos treatments after simulated colonic fermentation (Figure 6A). There was no difference between the treatments. Bacteroidetes relative abundance was around $2 \%$, compared to $5 \%$ in the control, while Firmicutes relative abundance remained the same as the blank and control. At the family level (Figure 6B), Fusobacteriaceae (22-30\%), Enterobacteriaceae (18-22\%), and Veillonellaceae $(22-30 \%)$ dominated in all four tibicos treatments. This differed 


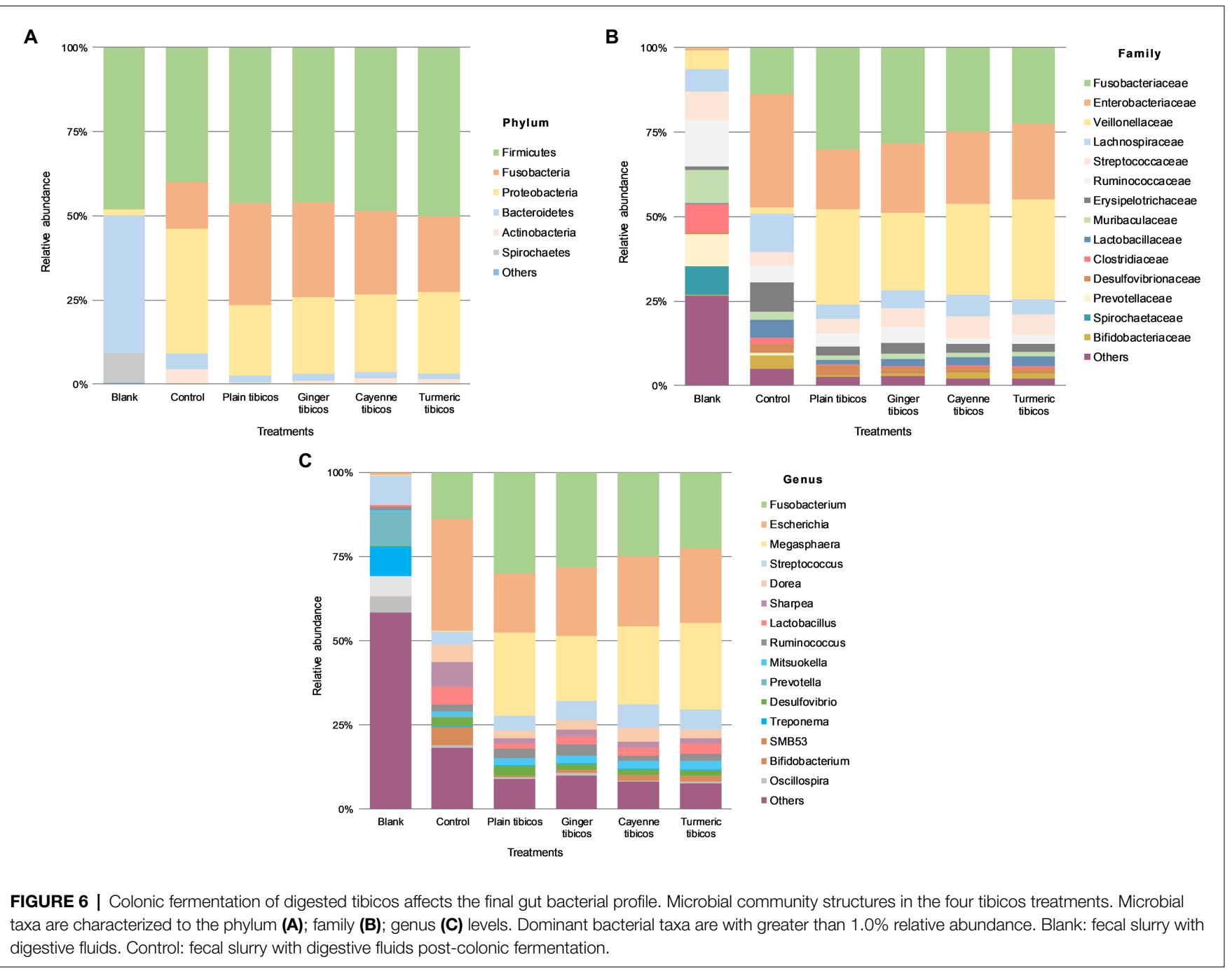

from the control, where Lachnospiraceae was more abundant than Veillonellaceae. When taxonomic composition was examined at genus level (Figure 6C), the dominant genus in all tibicos treatments was Fusobacterium at between 22 and 30\% relative abundance, twice as abundant as in the control. Escherichia relative abundance halved compared to the control, to around $20 \%$. There was no difference in relative abundance between the tibicos treatments. Compared to the control (0.4\%), compression of relative abundance with enrichment in Megasphaera (between 19 and 25\%) was observed. In the control, Lactobacillus relative abundance was approximately 5\%; in plain tibicos, it was reduced to $1.4 \%$, while the spice-added tibicos were between 2 and 3\%. Similarly, Bifidobacterium relative abundance was $4 \%$ in the control, while plain tibicos was reduced tenfold at $0.4 \%$; the spice-added treatments were between 0.8 and $1.6 \%$.

\section{DISCUSSION}

Botanical fermented food components are of increasing interest as potential beneficial modulators of gut microbiota for the management of metabolic noncommunicable diseases (Şanlier et al., 2019), but few studies focus on their application as whole foods from production to digestion. Our study showed that LAB and yeasts in sauerkraut and tibicos can be manipulated during production and storage for maximal microbial viability, may have higher survival rates when digested in their native whole food matrix, and can impact the relative abundance of gut bacteria.

\section{Storage Length and Starter Cultures Affect Microbial Viability of LAB and Yeasts}

The factors that impact LAB and yeast viability in both tibicos and sauerkraut are well studied. These include ingredient variation, salt concentration, use of starter cultures, and storage conditions, which affect metabolite concentration, $\mathrm{pH}$ and oxygen levels. A comprehensive review of tibicos microbial dynamics during production and storage has been performed by Lynch et al. (2021), Laureys and De Vuyst (2014), Laureys et al. (2017, 2018, 2019, 2021), and for sauerkraut, this area has been recently reviewed by Zabat et al. (2018) and Yang et al. (2020). 
In our work, the initial LAB population on the surface of shredded cabbage was around $3 \log \mathrm{cfu} / \mathrm{ml}$, which is within the typical range of fresh cabbage microflora (Fleming et al., 1988). As shown in Figure 1, inoculation with a starter culture labeled as containing $L$. plantarum, $L$. mesenteroides, and $P$. acidilactici caused an expected initial $2 \log$ increase in the LAB count. For both spontaneously fermented and inoculated sauerkraut, LAB counts peaked at 8-8.5 $\log \mathrm{cfu} / \mathrm{ml}$ on day 13 . A similar result was observed in $\mathrm{Lu}$ et al.'s (2003) study where LAB counts peaked at 8.4 $\log \mathrm{cfu} / \mathrm{ml}$ on day 15. However, Beganović et al. (2011) reported that inoculated sauerkraut required 21 days of fermentation to reach a peak of $7.63 \mathrm{log} \mathrm{cfu} / \mathrm{ml}$, and 28 days for spontaneously fermented sauerkraut (6.26 $\log \mathrm{cfu} / \mathrm{ml}$ ). These differences may be attributed to higher $4.0 \% \mathrm{NaCl}$ concentration, which inhibits the utilization of sucrose, reduces $\mathrm{LAB}$ metabolic rates, and leads to sauerkraut maturation delays (Xiong et al., 2016). The LAB counts of the spontaneously fermented sauerkrauts dropped to between 6 and $7 \log \mathrm{cfu} /$ $\mathrm{ml}$ by day 95 . This is typically seen in sauerkraut fermentations, due to the inhibitory effect of lactic acid and the gradual depletion of sugars (Xiong et al., 2012). In contrast, by day 95, inoculated sauerkraut LAB counts had dropped to undetectable levels. These results differ from those of Pandey and Garg (2015), who found that the LAB count of inoculated sauerkraut was consistently higher than that of spontaneously fermented sauerkraut. Conversely, Xiong et al. (2016) found that the desired rapid accumulation of lactic acid and subsequent low $\mathrm{pH}$ environment of inoculated sauerkraut may restrict $\mathrm{LAB}$ growth, leading to faster reduction in microbial proliferation. Commercial sauerkrauts are often fermented for 4-6 weeks, with a shelf life of up to 12 months. Our study established that, in order to maximize its probiotic potential and meet the requirements for probiotic foods (>6 $\log \mathrm{cfu} / \mathrm{ml}$ ), sauerkraut should be consumed within 7 weeks of manufacture.

In our study of tibicos, we were interested in understanding the effects of prolonged storage (96 days), as this is a typical shelf life of commercial tibicos. We were unable to find other studies of tibicos where storage exceeded 35 days. Our study observed the same trends as other tibicos studies (Miguel et al., 2011; Laureys and De Vuyst, 2014; Viana et al., 2017) in pH, metabolite production and carbohydrate depletion (Supplementary Material), and microbial counts during fermentation (Figure 2). Peak microbial counts during fermentation did differ between studies; this is likely due to tibicos grains from diverse geographic locations and the varied array of substrates used. In our study and those mentioned above, there was initial LAB growth with yeast suppression during initial fermentation. This was followed by an increase in yeast populations over 21 days, as LAB growth slowed but remained steady. Yeast counts in all treatments peaked at around $7 \log \mathrm{cfu} / \mathrm{ml}$ on day 35 . LAB counts of all treatments increased, reaching a peak of around $7 \mathrm{log} \mathrm{cfu} / \mathrm{ml}$ on different days: cayenne on day 19, plain on day 35 , and ginger and turmeric on day 47. These differences are likely due to the varying availability of spice-related nutrients during tibicos fermentation, which impact substrate consumption, metabolite concentrations, and microbial growth and species diversity (Laureys and de Vuyst, 2017; Laureys et al., 2018). Then followed a gradual decline in the viable counts of both yeasts and LAB, with all treatments except ginger tibicos at 6-6.5 $\log \mathrm{cfu} / \mathrm{ml}$ by day 96 . A 2016 study by Fiorda et al. (2016) on honey must kefir showed similar results, with yeast and LAB counts of 6-7 log $\mathrm{cfu} / \mathrm{ml}$ remaining stable until the end of their 35-day storage period. In our study, the LAB and yeast counts in ginger tibicos remained stable and were significantly higher than the other three treatments on day $96(p<0.05)$; this is discussed further below. Overall, we found that in order to ensure maximum total microbial counts at consumption, tibicos should be stored for around 28 days at $4^{\circ} \mathrm{C}$, but no longer.

\section{The Addition of Ginger Significantly Increased and Sustained Microbial Viability of LAB and Yeasts in Tibicos During Fermentation and Storage}

Spices are often used as functional ingredients and bio-preservatives in fermented foods, prized for their nutrient content, high antioxidant capacity, and aromatic flavor properties (Wahba et al., 2009). Ginger, cayenne, and turmeric possess anti-inflammatory properties, known to be beneficial for human health (Kunnumakkara et al., 2018). In our study, these spices were added on day 3 for a 48 -h secondary fermentation. The $\mathrm{pH}$ of the tibicos immediately increased, with ginger and turmeric tibicos $\mathrm{pH}$ remaining higher than plain and cayenne tibicos throughout storage (Supplementary Material); microbial counts continued upward following addition of spices (Figure 2). This is likely due to several factors that support microbial metabolism, such as the presence of fiber as a major substrate during secondary fermentation; bioavailable polyphenols; and spicerelated micronutrients, such as iron, copper, and amino acids. Çevik et al. (2019) showed that tibicos grains cultivated in honey or grape molasses solutions, both rich in vitamins and minerals, led to a significantly larger increase in LAB and yeast counts by the end of fermentation, compared to sucrose solution. However, in our study, by the end of secondary fermentation on day 5 , there was no significant difference between the $\mathrm{pH}$ and microbial counts of the four tibicos treatments.

Besides its medicinal uses, ginger ( $Z$. officinale) is often employed as a bio-preservative in the food industry due to its antifungal, antimicrobial, and antioxidant properties (Obi, 2015; Arekemase and Babashola, 2019). In the present study, only the addition of ginger significantly increased and sustained both LAB and yeast loads at approximately $7 \mathrm{log} \mathrm{cfu} / \mathrm{ml}(p<0.05)$ during prolonged storage, compared to plain, cayenne, and turmeric tibicos, whose microbial loads declined during the last month of storage (Figure 2). The accelerated growth of LAB in ginger tibicos in our study is consistent with Wang et al. (2017), whose study demonstrated that the addition of ginger extract to pickle fermentation increased LAB growth. However, other studies showed varying results, likely due to the application of different concentrations of ginger in a variety of substrates. Goharjoo et al. (2020) investigated the effect of different 
concentrations of ginger on $\mathrm{LAB}$ counts in fermented carrot juice and found that growth of $\mathrm{LAB}$ in a $4 \%$ treatment was significantly higher while the $\mathrm{pH}$ was lower than in 0 and $8 \%$ treatments $(p<0.01)$. This is likely due to the antibacterial nature of gingerols (Mahady et al., 2003; Rahmani et al., 2014). Our study observed that despite ginger tibicos having the highest levels of LAB, yeasts, and lactic acid, it reached the highest $\mathrm{pH}$ of 3.7. Laureys et al. (2018) showed that in a high nutrient environment, tibicos fermentation proceeds at a faster rate, resulting in high metabolite concentrations without a drop in $\mathrm{pH}$; this protects microbial growth from acidic stress. The $\mathrm{pH}$ of ginger tibicos then remained at around 3.3 throughout storage, while the $\mathrm{pH}$ of plain, cayenne, and turmeric tibicos fell below 3.3 (Supplementary Material). Silva et al. (2009) showed that when the $\mathrm{pH}$ of tibicos falls under 3.3, microbial activity is restricted due to acidity pressure. The growth and maintenance of yeast in concert with LAB concurs with findings of Stadie et al. (2013) regarding their mutualistic relationship. Laureys et al. (2018) showed that nutrient concentration during fermentation is associated with shifts in dominant microbial species, with high nutrient concentrations favoring $L$. nagelii and S. cerevisiae.

We note here that in our study, due to consumer-based flavor considerations, spice concentrations varied. Although ginger, at the highest concentration of $0.5 \%$, showed significantly higher LAB and yeast growth, turmeric $(0.25 \%)$ had a lower microbial load than cayenne $(0.125 \%)$, which was not significantly different to plain tibicos. The complexity of whole food ingredients is such that equal concentrations of different spices do not contain the same concentrations of nutrients; also, each spice contains unique bioactive components that have varying effects on microbial growth (Liu et al., 2017).

\section{LAB Survival in a Simulated Upper Gastrointestinal Tract Is Significantly Improved by the Addition of Ginger and Cayenne to Tibicos}

Though spices have been found to affect fermentation and storage, they may also have significant protective effects during digestion. The mechanisms are likely related to their antioxidant, antimicrobial, and prebiotic properties. A 2019 human randomized placebocontrolled pilot study by Lu et al. (2019) showed that consumption of a spice mixture, which included ginger and cayenne, resulted in significant modification of gut microbiota due to spices' prebiotic effects. In our study, the addition of cayenne and ginger significantly improved LAB counts $(p<0.05)$ compared to plain tibicos, while the viability of yeasts was not affected (Figure 4). However, cayenne LAB survival rates (Table 2) were significantly higher at $82 \%(p<0.05)$ than ginger $(77 \%)$, plain $(74 \%)$, and turmeric $(73 \%)$. Cayenne and plain tibicos had (80\%) significantly higher $(p<0.05)$ yeast survival rates than ginger $(78 \%)$ and turmeric (77\%). Antioxidant bioavailability and activity is increased by the gastrointestinal environment, as shown in simulated gastric and duodenal digestion studies of various fruits and vegetables (Pérez-Vicente et al., 2002; Bouayed et al., 2011). Valero-Cases et al. (2017) attributed high microbial survival in fermented pomegranate juice to the increased bio-accessibility of antioxidant phenolic compounds before in vitro digestion, enhancing the ability of LAB to survive in vitro digestion. The prebiotic effects and high antioxidant capacity of spices such as ginger and cayenne may synergistically improve tibicos microbial survival in a simulated digestive environment. Our study suggests that microbes in tibicos can withstand digestive fluids and low $\mathrm{pH}$, and that added spices may improve microbial viability.

\section{When Digested as Part of Naturally Produced Whole Fermented Foods, LAB and Yeasts Have High Survival Rates}

Probiotic microorganisms must be able to endure the adversity of gastrointestinal travel, from salivary enzymes, to gastric acids, bile acids, and pancreatic juices (Food and Agriculture Organization of the United Nations and World Health Organisation, 2002; Derrien and van Hylckama Vlieg, 2015). Being naturally protected by their coevolved food matrices may assist in their effective transport to the large intestine. To our knowledge, the present study is the first to apply sauerkraut and tibicos in their whole food form to a simulated digestion model. Our aim was to investigate whether microbes in fermented foods were better able to survive the digestive tract when protected by their established food matrices.

In all four sauerkraut treatments, LAB counts fell significantly after in vitro digestion (Figure $3 ; p<0.05$ ). Salt concentration and use of starter cultures did not affect $L A B$ survival after simulated digestion. LAB counts of all sauerkraut treatments experienced an approximate 2 log loss. Overall, approximately 6 out of $8 \log \mathrm{cfu} / \mathrm{ml}$ of LAB survived GI simulation, with a microbial survival rate of around $72 \%$ (Table 1). This two-log unit loss of $\mathrm{LAB}$ concurred with whole food in vitro digestion studies of other fermented foods; for example, LAB-fermented milks (Faye et al., 2012) and fermented vegetable juices (Değirmencioğlu et al., 2016). These microbial survival rates may be ascribed to low sensitivity to acidic $\mathrm{pH}$, high hydrophobicity, and low sensitivity to bile salts (Zanirati et al., 2015). In contrast, Beganović et al. (2014) observed that 25 LAB strains isolated from sauerkraut and then applied as a pure culture without the food matrix underwent a 3-5 log loss (from $9 \log \mathrm{cfu} / \mathrm{ml}$ to between 4 and $6 \mathrm{log} \mathrm{cfu} / \mathrm{ml}$ ) post-in vitro digestion. This indicates that $\mathrm{LAB}$ delivered in a sauerkraut whole food matrix may be further protected from the GI

TABLE 2 | The addition of cayenne powder to tibicos significantly increases lactic acid bacteria (LAB) survival rate during in vitro digestion.

\begin{tabular}{lcc}
\hline Treatments & LAB survival rate (\%) & Yeast survival rate (\%) \\
\hline Plain tibicos & $74.5 \pm 2.6^{\mathrm{ac}}$ & $80.2 \pm 0.7^{\mathrm{ac}}$ \\
Ginger tibicos & $77.0 \pm 0.7^{\mathrm{a}}$ & $77.8 \pm 0.6^{\mathrm{bd}}$ \\
Cayenne tibicos & $81.6 \pm 2.0^{\mathrm{b}}$ & $80.3 \pm 0.2^{\mathrm{a}}$ \\
Turmeric tibicos & $73.2 \pm 2.1^{\mathrm{c}}$ & $77.3 \pm 1.1^{\text {de }}$ \\
\hline
\end{tabular}

Percentage survival of tibicos-associated $L A B$ after simulated gastric and small intestinal digestion. Means within a column with different superscripts (a-c) are significantly different $(p<0.05)$. The results expressed as mean $(n=3) \pm S D$ (standard deviation). 
environment by their natural incorporation with suitable food substrates. As such, sauerkraut may be an appropriate matrix for the delivery of probiotic strains, whether inoculated or spontaneously fermented.

Our study of tibicos showed significant microbial losses after simulated digestion $(p<0.05$; Figure 4$)$, with a one to two $\log$ unit loss of LAB and yeast after the small intestinal phase. The LAB survival rate was around $73-82 \%$, while yeast survival was around $77-80 \%$ (Table 2). Değirmencioğlu et al. (2016) conducted an in vitro digestion study with fermented pomegranate juice containing LAB and two Saccharomyces yeasts commonly found in tibicos. They found that, depending on the yeast involved, LAB survival rates were between 67 and $73 \%$ (S. cerevisiae) and 74 and $77 \%$ (S. boulardii); our study had similar findings. However, when it comes to yeast survival, our rates were lower than those found in Değirmencioğlu et al.s study (92-98\%). This indicates that $\mathrm{LAB}$ and yeast survive digestion in whole food matrices. In regard to in vitro survival of strains isolated from tibicos, Romero-Luna et al. (2020) found that L. casei CT12 extracted from tibicos grains had a $40 \%$ survival rate after in vitro digestion (2020); another of their studies (2019) found that $S$. cerevisiae C41 isolated from tibicos had a $78 \%$ survival rate post-in vitro digestion. A 2019 study by Angelescu et al. (2019), L. plantarum CR1 isolated from tibicos had a survival rate of $79 \%$ after in vitro digestion. These varying results may be due to heterogeneous methodologies. The needs of microbes are strain specific, and while some may benefit from integration into a food matrix-based microbial community, others may not. Sequencing of the microbial communities throughout fermentation, storage and simulated digestion would help ascertain which species of LAB and yeasts may benefit from being integrated in a whole food matrix.

\section{Whole Digested Tibicos and Sauerkraut Change Composition and Relative Abundance of Bacteria During Colonic Fermentation}

Despite the rapid increase in studies focused on the interaction of isolated fermented food components and microorganisms with the gut microbiome, these complex interactions are still poorly understood (Douillard and de Vos, 2019).

In our study, bacterial relative abundance and composition in the control and digested fermented food samples were affected by in vitro colonic fermentation in a similar way, due to the resident colonic microbiota in the feces (Aguirre and Venema, 2017). Firmicutes, Fusobacteria, and Proteobacteria were the dominant phyla in all samples (Figures 5, 6). However, there were some marked differences in relative abundance between the control and the fermented food samples. Bacteroidetes abundance dropped in tibicos and sauerkraut samples, while Firmicutes abundance increased or remained at a similar level to the control (Figures 5A, 6A). This finding was similar to other in vitro studies of the impact of probiotic goat milk with passionfruit by-product (Casarotti et al., 2020) and snow chrysanthemum polysaccharides (Wu et al., 2021b) on gut microbiota. In vivo studies in mice with LAB-fermented goat milk (Chen et al., 2020) and kefir (Hsu et al., 2018) also observed an increased Firmicutes to Bacteroidetes (F/B) ratio in the treatment group. On the other hand, other polysaccharides, from okra (Wu et al., 2021c), flaxseed (Zhou et al., 2020), and mushrooms (Liu et al., 2021), observed increased Bacteroidetes abundance, and reduced Firmicutes, with a lower F/B ratio. Polyphenols act as prebiotics in the gut and have also been shown to influence $\mathrm{F} / \mathrm{B}$ ratio through the inhibition of certain bacterial species (Loo et al., 2020). This indicates that changes in gut microbiota are dependent on the substrates involved. F/B ratio must be considered with the abundance of particular bacterial species, the interplay with microbial diversity and the complexity of individual contributing factors, e.g., host genetics, baseline microbiota, and comorbidities.

Compared to the blank and the control, colonic fermentation of all digested sauerkraut and tibicos samples led to an up to 85-fold increase in Megasphaera, a genus of Firmicutes bacteria (Figures 5C, 6C). Megasphaera sp. have been found to have important metabolic roles in the human and porcine gut microbiome: $M$. eldensii, NM10 and BL7 produce essential amino acids and vitamins in the gut (Shetty et al., 2013) and utilize ruminal lactate to produce the beneficial short-chain fatty acid, butyrate (Hashizume et al., 2003; Kim et al., 2011). Polysaccharides have been found to encourage Megasphaera growth during in vitro fecal fermentation (Wu et al., 2021a,b,c). In our study, it is likely that indigestible polysaccharides from tibicos and sauerkraut were available in sufficient quantities during colonic fermentation to encourage Megasphaera growth.

Salt concentration and inoculation of sauerkraut did not have an observable effect on relative abundance of gut microbiota (Figure 5). However, the unfermented cabbage with $1.5 \%$ salt treatment had differing relative abundances compared to the control and sauerkraut samples. Colonic fermentation of the unfermented cabbage led to an expansion of Enterobacter $(0-38 \%)$ as the dominant genus, with lower abundances of Escherichia, Fusobacterium, and Megasphaera than the sauerkraut. Lactobacillus and Bifidobacterium relative abundance was lower than the control but were two times and 10 times higher, respectively, than the sauerkraut samples. During the early stages of sauerkraut fermentation, Enterobacteriaceae levels in the food matrix have been shown to remain high (Lavefve et al., 2021). Our study, concurrent with others, observed increasing $\mathrm{LAB}$ counts during fermentation, with a peak at around 13 days. This suggests that the unfermented cabbage is undergoing an early fermentation process in the simulated colon, leading to domination by Enterobacter and rapid $\mathrm{LAB}$ growth. These findings suggest that the impact of fermentation and storage on microbial counts is one of the major reasons for differences in bacterial relative abundance at the gut level. As we did not measure fiber content throughout our study, it is difficult to assess whether this is simply due to the prebiotic effect of unfermented fiber from the cabbage. Other possible mechanisms driving these differences include the microbial transformation of substrates prior to consumption and ingested microbes, affecting the bioavailability of polysaccharides and phenolic compounds in the digestive tract. 
Currently, studying microbial communities in whole fermented foods has its drawbacks, in that only specific isolated strains can be further assessed for other prerequisites of probiotic potential and safety. A better understanding of the interplay between substrates and microbes in whole fermented foods requires sequencing of microbes, and monitoring of phenolic compounds, polysaccharides and microbial metabolites (including short-chain fatty acids), during fermentation, storage, and in vitro digestion. Although the harmonized static in vitro digestive system we used is well accepted in the study of food (LucasGonzález et al., 2018), it has its limitations as an in vivo substitute (Nissen et al., 2020). Complex, multi-omics dynamic GI digestion and fermentation models would allow deeper investigation of how fermented food components interact, and how they may elicit shifts in the diversity, abundance, and richness of core microbial communities over time (Nissen et al., 2020; Ji et al., 2021).

The present study showed that storage affects microbial survival in both low-salt sauerkraut and tibicos, with excessive storage having a slight but significant detrimental impact on LAB and yeast counts. This indicates that tibicos should be consumed within 28 days, while sauerkraut should be stored no longer than 7 weeks. LAB and yeasts in both foods are able to adequately survive the low $\mathrm{pH}$ environment of fermentation, with resulting microbial counts high enough to be considered probiotic. Ginger and cayenne were observed to significantly enhance LAB survival during fermentation, storage, and GI digestion, likely due to inherent prebiotics, such as fiber, and antioxidant capacity. Our in vitro digestion results suggest that both sauerkraut and tibicos contain acid, enzyme, and bile tolerant LAB and yeasts, some of which have better survival rates when consumed in a whole food matrix. Sauerkraut and tibicos LAB and yeasts were shown to shift composition and relative abundance of gut microbiota. In relation to their survival in the GI tract and effects on human health, dynamic in vitro models, human cell studies, and clinical trials are required.

\section{REFERENCES}

Aguirre, M., and Venema, K. (2017). Challenges in simulating the human gut for understanding the role of the microbiota in obesity. Benef. Microbes 8, 31-53. doi: 10.3920/BM2016.0113

Angelescu, I. R., Zamfir, M., Stancu, M. M., and Grosu-Tudor, S.-S. (2019). Identification and probiotic properties of lactobacilli isolated from two different fermented beverages. Ann. Microbiol. 69, 1557-1565. doi: 10.1007/ s13213-019-01540-0

Arekemase, M. O., and Babashola, D. R. (2019). Assessment of the effectiveness of ginger (Zingiber officinale), clove (Syzygium aromaticum) and sodium benzoate on the shelf life of soymilk. Not. Sci. Biol. 11, 400-409. doi: 10.15835/nsb11410462

Beganović, J., Kos, B., Leboš Pavunc, A., Uroić, K., Jokić, M., and Šušković, J. (2014). Traditionally produced sauerkraut as source of autochthonous functional starter cultures. Microbiol. Res. 169, 623-632. doi: 10.1016/j. micres.2013.09.015

Beganović, J., Pavunc, A. L., Gjuračić, K., Špoljarec, M., Šušković, J., and Kos, B. (2011). Improved sauerkraut production with probiotic strain Lactobacillus plantarum L4 and Leuconostoc mesenteroides LMG 7954. J. Food Sci. 76, M124-M129. doi: 10.1111/j.1750-3841.2010.02030.x

Bouayed, J., Hoffmann, L., and Bohn, T. (2011). Total phenolics, flavonoids, anthocyanins and antioxidant activity following simulated gastro-intestinal

\section{DATA AVAILABILITY STATEMENT}

The datasets presented in this study can be found in online repositories. The names of the repository/repositories and accession number(s) can be found at: https://www.ncbi.nlm. nih.gov/, PRJNA754823.

\section{AUTHOR CONTRIBUTIONS}

MC and $\mathrm{KH}$ conceived and designed the study. FY, YW, and DL carried out the laboratory experiments and analyzed the data with MC. MC wrote the manuscript, which was edited by KH. All authors contributed to the article and approved the submitted version.

\section{FUNDING}

MC was supported by an Australian Government Research Training Program (RTP) Scholarship through the University of Melbourne, Australia.

\section{ACKNOWLEDGMENTS}

The authors would like to thank the School of Agriculture and Food at The University of Melbourne for providing the laboratory resources for this project.

\section{SUPPLEMENTARY MATERIAL}

The Supplementary Material for this article can be found online at: https://www.frontiersin.org/articles/10.3389/fmicb.2021.759708/ full\#supplementary-material

digestion and dialysis of apple varieties: bioaccessibility and potential uptake. Food Chem. 128, 14-21. doi: 10.1016/j.foodchem.2011.02.052

Buriti, F. C. A., Castro, I. A., and Saad, S. M. I. (2010). Viability of Lactobacillus acidophilus in synbiotic guava mousses and its survival under in vitro simulated gastrointestinal conditions. Int. J. Food Microbiol. 137, 121-129. doi: 10.1016/j.ijfoodmicro.2009.11.030

Casarotti, S. N., Borgonovi, T. F., Tieghi, T. D., Sivieri, K., and Penna, A. L. (2020). Probiotic low-fat fermented goat milk with passion fruit by-product: in vitro effect on obese individuals' microbiota and on metabolites production. Food Res. Int. 136:109453. doi: 10.1016/j.foodres.2020.109453

Caporaso, J. G., Kuczynski, J., Stombaugh, J., Bittinger, K., Bushman, F. D., Costello, E. K., et al. (2010). QIIME allows analysis of high-throughput community sequencing data. Nat. Methods. 7:335. doi: 10.1038/ nmeth.f.303

Çevik, T., Aydoğdu, N. S., Özdemir, N., and Taş, T. (2019). The effect of different sugars on water kefir grains. Turk. J. Agric. Food Sci. Technol. 7, 40-45. doi: 10.24925/turjaf.v7isp1.40-45.2687

Chen, X., Zheng, R., Liu, R., and Li, L. (2020). Goat milk fermented by lactic acid bacteria modulates small intestinal microbiota and immune responses. J. Funct. Foods 65:103744. doi: 10.1016/j.jff.2019.103744

De Angelis, M., Campanella, D., Cosmai, L., Summo, C., Rizzello, C. G., and Caponio, F. (2015). Microbiota and metabolome of un-started and started 
Greek-type fermentation of Bella Di Cerignola table olives. Food Microbiol. 52, 18-30. doi: 10.1016/j.fm.2015.06.002

DeSantis, T. Z., Hugenholtz, P., Larsen, N., Rojas, M., Brodie, E. L., Keller, K., et al. (2006). Greengenes, a chimera-checked 16S rRNA gene database and workbench compatible with ARB. Appl. Environ. Microbiol. 72, 5069-5072. doi: 10.1128/AEM.03006-05

Değirmencioğlu, N., Gurbuz, O., and Şahan, Y. (2016). The monitoring, via an in vitro digestion system, of the bioactive content of vegetable juice fermented with Saccharomyces cerevisiae and Saccharomyces boulardii. J. Food Process. Preserv. 40, 798-811. doi: 10.1111/jfpp.12704

Derrien, M., and van Hylckama Vlieg, J. E. T. (2015). Fate, activity, and impact of ingested bacteria within the human gut microbiota. Trends Microbiol. 23, 354-366. doi: 10.1016/j.tim.2015.03.002

Di Cagno, R., Coda, R., De Angelis, M., and Gobbetti, M. (2013). Exploitation of vegetables and fruits through lactic acid fermentation. Food Microbiol. 33, 1-10. doi: 10.1016/j.fm.2012.09.003

Douillard, F. P., and de Vos, W. M. (2019). Biotechnology of health-promoting bacteria. Biotechnol. Adv. 37:107369. doi: 10.1016/j.biotechadv.2019.03.008

Edgar, R. C. (2010). Search and clustering orders of magnitude faster than BLAST. Bioinformatics 26, 2460-2461. doi: 10.1093/bioinformatics/btq461

Faye, T., Tamburello, A., Vegarud, G. E., and Skeie, S. (2012). Survival of lactic acid bacteria from fermented milks in an in vitro digestion model exploiting sequential incubation in human gastric and duodenum juice. J. Dairy Sci. 95, 558-566. doi: 10.3168/jds.2011-4705

Fiorda, F. A., de Melo Pereira, G. V., Thomaz-Soccol, V., Rakshit, S. K., and Soccol, C. R. (2016). Evaluation of a potentially probiotic non-dairy beverage developed with honey and kefir grains: fermentation kinetics and storage study. Food Sci. Technol. Int. 22, 732-742. doi: 10.1177/1082013216646491

Fischer, M. M., Egli, I. M., Aeberli, I., Hurrell, R. F., and Meile, L. (2014). Phytic acid degrading lactic acid bacteria in tef-injera fermentation. Int. J. Food Microbiol. 190, 54-60. doi: 10.1016/j.ijfoodmicro.2014.08.018

Fleming, H. P., McFeeters, R. F., and Humphries, E. G. (1988). A fermentor for study of sauerkraut fermentation. Biotechnol. Bioeng. 31, 189-197. doi: 10.1002/bit.260310302

Food and Agriculture Organization of the United Nations and World Health Organisation (2002). Guidelines for the evaluation of probiotics in food. Available at: https://www.who.int/foodsafety/fs_management/en/probiotic guidelines.pdf (Accessed August 15, 2020).

Forssten, S. D., Sindelar, C. W., and Ouwehand, A. C. (2011). Probiotics from an industrial perspective. Anaerobe 17, 410-413. doi: 10.1016/j.anaerobe. 2011.04.014

Gbassi, G. K., Vandamme, T., Yolou, F. S., and Marchioni, E. (2011). In vitro effects of $\mathrm{pH}$, bile salts and enzymes on the release and viability of encapsulated Lactobacillus plantarum strains in a gastrointestinal tract model. Int. Dairy J. 21, 97-102. doi: 10.1016/j.idairyj.2010.09.006

Gille, D., Schmid, A., Walther, B., and Vergères, G. (2018). Fermented food and non-communicable chronic diseases: a review. Nutrients 10:448. doi: 10.3390/nu10040448

Goharjoo, M. E., Dovom, M. R., Shahidi, F., Yazdi, F. T., and Varidi, M. (2020). Evaluation of the ginger and yogurt serum different levels on the lactic flora biodiversity in fermented carrot. J. Food Process. Preserv. 45:e15111. doi: $10.1111 /$ jfpp.15111

Hashizume, K., Tsukahara, T., Yamada, K., Koyama, H., and Ushida, K. (2003). Megasphaera elsdenii JCM1772T normalizes hyperlactate production in the large intestine of fructooligosaccharide-fed rats by stimulating butyrate production. J. Nutr. 133, 3187-3190. doi: 10.1093/jn/133.10.3187

Hernandez-Hernandez, O., Muthaiyan, A., Moreno, F. J., Montilla, A., Sanz, M. L., and Ricke, S. C. (2012). Effect of prebiotic carbohydrates on the growth and tolerance of Lactobacillus. Food Microbiol. 30, 355-361. doi: 10.1016/j. fm.2011.12.022

Hsu, Y. J., Huang, W. C., Lin, J. S., Chen, Y. M., Ho, S. T., Huang, C. C., et al. (2018). Kefir supplementation modifies gut microbiota composition, reduces physical fatigue, and improves exercise performance in mice. Nutrients 10:862. doi: $10.3390 /$ nu10070862

Ji, H., Hu, J., Zuo, S., Zhang, S., Li, M., and Nie, S. (2021). In vitro gastrointestinal digestion and fermentation models and their applications in food carbohydrates. Crit. Rev. Food Sci. Nutr., 1-23. doi: 10.1080/10408398.2021.1884841
Kim, H. B., Borewicz, K., White, B. A., Singer, R. S., Sreevatsan, S., Tu, Z. J., et al. (2011). Longitudinal investigation of the age-related bacterial diversity in the feces of commercial pigs. Vet. Microbiol. 153, 124-133. doi: 10.1016/j. vetmic.2011.05.021

Kunnumakkara, A. B., Sailo, B. L., Banik, K., Harsha, C., Prasad, S., Gupta, S. C., et al. (2018). Chronic diseases, inflammation, and spices: how are they linked? J. Transl. Med. 16:14. doi: 10.1186/s12967-018-1381-2

La Anh, N. (2015). Health-promoting microbes in traditional vietnamese fermented foods: a review. Food Sci. Human Wellness 4, 147-161. doi: 10.1016/j.fshw.2015.08.004

Lara-Hidalgo, C. E., Hernandez-Sanchez, H., Hernandez-Rodriguez, C., and Dorantes-Alvarez, L. (2017). Yeasts in fermented foods and their probiotic potential. Austin J. Nutr. Metab. 4:1045

Laureys, D., Aerts, M., Vandamme, P., and De Vuyst, L. (2018). Oxygen and diverse nutrients influence the water kefir fermentation process. Food Microbiol. 73, 351-361. doi: 10.1016/j.fm.2018.02.007

Laureys, D., Aerts, M., Vandamme, P., and De Vuyst, L. (2019). The buffer capacity and calcium concentration of water influence the microbial species diversity, grain growth, and metabolite production during water kefir fermentation. Front. Microbiol. 10:2876. doi: 10.3389/fmicb.2019.02876

Laureys, D., and De Vuyst, L. (2014). Microbial species diversity, community dynamics, and metabolite kinetics of water kefir fermentation. Appl. Environ. Microbiol. 80, 2564-2572. doi: 10.1128/AEM.03978-13

Laureys, D., and De Vuyst, L. (2017). The water kefir grain inoculum determines the characteristics of the resulting water kefir fermentation process. J. Appl. Microbiol. 122, 719-732. doi: 10.1111/jam.13370

Laureys, D., Leroy, F., Hauffman, T., Raes, M., Aerts, M., Vandamme, P., et al. (2021). The type and concentration of inoculum and substrate as well as the presence of oxygen impact the water kefir fermentation process. Front. Microbiol. 12:628599. doi: 10.3389/fmicb.2021.628599

Laureys, D., Van Jean, A., Dumont, J., and De Vuyst, L. (2017). Investigation of the instability and low water kefir grain growth during an industrial water kefir fermentation process. Appl. Microbiol. Biotechnol. 101, 2811-2819. doi: 10.1007/s00253-016-8084-5

Lavefve, L., Cureau, N., Rodhouse, L., Marasini, D., Walker, L. M., and Ashley, D. (2021). Microbiota profiles and dynamics in fermented plant-based products and preliminary assessment of their in vitro gut microbiota modulation. Food Front. 2, 268-281. doi: 10.1002/fft2.75

Leech, J., Cabrera-Rubio, R., Walsh, A. M., Macori, G., Walsh, C. J., Barton, W., et al. (2020). Fermented-food metagenomics reveals substrate-associated differences in taxonomy and health-associated and antibiotic resistance determinants. mSystems 5:e00522-e00520. doi: 10.1128/mSystems.00522-20

Liu, Y., Li, Y., Ke, Y., Li, C., Zhang, Z., Wu, Y., et al. (2021). In vitro salivagastrointestinal digestion and fecal fermentation of Oudemansiella radicata polysaccharides reveal its digestion profile and effect on the modulation of the gut microbiota. Carbohydr. Polym. 251:117041. doi: 10.1016/j. carbpol.2020.117041

Liu, Q., Meng, X., Li, Y., Zhao, C. N., Tang, G. Y., and Li, H. B. (2017). Antibacterial and antifungal activities of spices. Int. J. Mol. Sci. 18:1283. doi: 10.3390/ijms18061283

London, L. E., Kumar, A. H., Wall, R., Casey, P. G., O’Sullivan, O., Shanahan, F., et al. (2014). Exopolysaccharide-producing probiotic lactobacilli reduce serum cholesterol and modify enteric microbiota in ApoE-deficient mice. J. Nutr. 144, 1956-1962. doi: 10.3945/jn.114.191627

Loo, Y. T., Howell, K., Chan, M., Zhang, P., and Ng, K. (2020). Modulation of the human gut microbiota by phenolics and phenolic fiber-rich foods. Compr. Rev. Food Sci. Food Saf. 19, 1268-1298. doi: 10.1111/1541-4337.12563

Lu, Q. Y., Rasmussen, A. M., Yang, J., Lee, R. P., Huang, J., Shao, P., et al. (2019). Mixed spices at culinary doses have prebiotic effects in healthy adults: a pilot study. Nutrients 11:1425. doi: 10.3390/nu11061425

Lucas-González, R., Viuda-Martos, M., Pérez-Alvarez, J. A., and Fernández-López, J. (2018). In vitro digestion models suitable for foods: opportunities for new fields of application and challenges. Food Res. Int. 107, 423-436. doi: 10.1016/j. foodres.2018.02.055

Lynch, K. M., Wilkinson, S., Daenen, L., and Arendt, E. K. (2021). An update on water kefir: microbiology, composition and production. Int. J. Food Microbiol. 345:109128. doi: 10.1016/j.ijfoodmicro.2021.109128 
Mahady, G. B., Pendland, S. L., Yun, G. S., Lu, Z.-Z., and Stoia, A. (2003). Ginger (Zingiber officinale Roscoe) and the gingerols inhibit the growth of cag A+ strains of helicobacter pylori. Anticancer Res. 23, 3699-3702

Mäkinen, O., Wanhalinna, V., Zannini, E., and Arendt, E. K. (2016). Foods for special dietary needs: non-dairy plant-based milk substitutes and fermented dairy-type products. Crit. Rev. Food Sci. Nutr. 56, 339-349. doi: 10.1080/10408398.2012.761950

Marco, M. L., Heeney, D., Binda, S., Cifelli, C. J., Cotter, P. D., Foligné, B., et al. (2017). Health benefits of fermented foods: microbiota and beyond. Curr. Opin. Biotechnol. 44, 94-102. doi: 10.1016/j.copbio.2016.11.010

Marsh, A. J., O'Sullivan, O., Hill, C., Ross, R. P., and Cotter, P. D. (2013). Sequence-based analysis of the microbial composition of water kefir from multiple sources. FEMS Microbiol. Lett. 348, 79-85. doi: 10.1111/15746968.12248

Miguel, M. G. C. P., Cardoso, P. G., Magalhães, K. T., and Schwan, R. F. (2011). Profile of microbial communities present in tibico (sugary kefir) grains from different Brazilian states. World J. Microbiol. Biotechnol. 27, 1875-1884. doi: 10.1007/s11274-010-0646-6

Minekus, M., Alminger, M., Alvito, P., Ballance, S., Bohn, T., Bourlieu, C., et al. (2014). A standardised static in vitro digestion method suitable for food - an international consensus. Food Funct. 5, 1113-1124. doi: 10.1039/ C3FO60702J

Nissen, L., Casciano, F., and Gianotti, A. (2020). Intestinal fermentation in vitro models to study food-induced gut microbiota shift: an updated review. FEMS Microbiol. Lett. 367:fnaa097. doi: 10.1093/femsle/fnaa097

Obi, C. D. (2015). Assessment of the preservative effects of different local spices and their flavor acceptability in Hibiscus sabdariffa calyx drinks. Int. J. Agric. Rural. Dev. 18, 2161-2165.

Pandey, S., and Garg, F. C. (2015). Preparation of spiced sauerkraut by using lactic acid bacteria and by natural fermentation. Int. J. Sci. Res. 4, 2753-2761.

Pasolli, E., De Filippis, F., Mauriello, I. E., Cumbo, F., Walsh, A. M., Leech, J., et al. (2020). Large-scale genome-wide analysis links lactic acid bacteria from food with the gut microbiome. Nat. Commun. 11:2610. doi: 10.1038/ s41467-020-16438-8

Pérez-Vicente, A., Gil-Izquierdo, A., and García-Viguera, C. (2002). In vitro gastrointestinal digestion study of pomegranate juice phenolic compounds, anthocyanins, and vitamin C. J. Agric. Food Chem. 50, 2308-2312. doi: $10.1021 / \mathrm{jf} 0113833$

Perrin, S., Grill, J. P., and Schneider, F. (2000). Effects of fructooligosaccharides and their monomeric components on bile salt resistance in three species of bifidobacteria. J. Appl. Microbiol. 88, 968-974. doi: 10.1046/j.1365-2672. 2000.01070.x

Rahmani, A. H., Shabrmi, F. M., and Aly, S. M. (2014). Active ingredients of ginger as potential candidates in the prevention and treatment of diseases via modulation of biological activities. Int. J. Physiol. Pathophysiol. Pharmacol. $6,125-136$

Ranadheera, R. D. C. S., Baines, S. K., and Adams, M. C. (2010). Importance of food in probiotic efficacy. Food Res. Int. 43, 1-7. doi: 10.1016/j. foodres.2009.09.009

Romero-Luna, H. E., Hernández-Sánchez, H., and Dávila-Ortiz, G. (2017). Traditional fermented beverages from Mexico as a potential probiotic source. Ann. Microbiol. 67, 577-586. doi: 10.1007/s13213-017-1290-2

Romero-Luna, H. E., Hernández-Sánchez, H., Ribas-Aparicio, R. M., Cauich-Sánchez, P. I., and Dávila-Ortiz, G. (2019). Evaluation of the probiotic potential of Saccharomyces cerevisiae strain (C41) isolated from Tibicos by in vitro studies. Probiotics Antimicrob. Proteins 11, 794-800. doi: 10.1007/ s12602-018-9471-2

Romero-Luna, H. E., Peredo-Lovillo, A., Hernández-Mendoza, A., Hernández-Sánchez, H., Cauich-Sánchez, P. I., Ribas-Aparicio, R. M., et al. (2020). Probiotic potential of Lactobacillus paracasei CT12 isolated from water kefir grains (Tibicos). Curr. Microbiol. 77, 2584-2592. doi: 10.1007/ s00284-020-02016-0

Şanlier, N., Gökcen, B. B., and Sezgin, A. C. (2019). Health benefits of fermented foods. Crit. Rev. Food Sci. Nutr. 59, 506-527. doi: 10.1080/10408398. 2017.1383355

Septembre-Malaterre, A., Remize, F., and Poucheret, P. (2018). Fruits and vegetables, as a source of nutritional compounds and phytochemicals: changes in bioactive compounds during lactic fermentation. Food Res. Int. 104, 86-99. doi: $10.1016 /$ j.foodres.2017.09.031
Shetty, S. A., Marathe, N. P., Lanjekar, V., Ranade, D., and Shouche, Y. S. (2013). Comparative genome analysis of Megasphaera sp. reveals niche specialization and its potential role in the human gut. PLoS One 8:e79353. doi: 10.1371/journal.pone.0079353

Silva, K. R., Rodrigues, S. A., Filho, L. X., and Lima, Á. S. (2009). Antimicrobial activity of broth fermented with kefir grains. Appl. Biochem. Biotechnol. 152, 316-325. doi: 10.1007/s12010-008-8303-3

Sirisena, S., Ajlouni, S., and Ng, K. (2018). Simulated gastrointestinal digestion and in vitro colonic fermentation of date (Phoenix dactylifera L.) seed polyphenols. Int. J. Food Sci. Technol. 53, 412-422. doi: 10.1111/ijfs.13599

Stadie, J., Gulitz, A., Ehrmann, M. A., and Vogel, R. F. (2013). Metabolic activity and symbiotic interactions of lactic acid bacteria and yeasts isolated from water kefir. Food Microbiol. 35, 92-98. doi: 10.1016/j. fm.2013.03.009

Su, P., Henriksson, A., and Mitchell, H. (2007). Prebiotics enhance survival and prolong the retention period of specific probiotic inocula in an in vivo murine model. J. Appl. Microbiol. 103, 2392-2400. doi: 10.1111/j.1365-2672. 2007.03469.x

Tamang, J. P., Watanabe, K., and Holzapfel, W. H. (2016). Review: diversity of microorganisms in global fermented foods and beverages. Front. Microbiol. 7:377. doi: $10.3389 /$ fmicb.2016.00377

Tsitko, I., Wiik-Miettinen, F., Mattila, O., Rosa-Sibakov, N., Seppänen-Laakso, T., Maukonen, J., et al. (2019). A small in vitro fermentation model for screening the gut microbiota effects of different fiber preparations. Int. J. Mol. Sci. 20:1925. doi: $10.3390 /$ ijms 20081925

Valero-Cases, E., Nuncio-Jáuregui, N., and Frutos, M. J. (2017). Influence of fermentation with different lactic acid bacteria and in vitro digestion on the biotransformation of phenolic compounds in fermented pomegranate juices. J. Agric. Food Chem. 65, 6488-6496. doi: 10.1021/acs.jafc.6b04854

Viana, R. O., Magalhães-Guedes, K. T., Braga, R. A., Dias, D. R., and Schwan, R. F. (2017). Fermentation process for production of apple-based kefir vinegar: microbiological, chemical and sensory analysis. Braz. J. Microbiol. 48, 592-601. doi: $10.1016 /$ j.bjm.2016.11.006

Wahba, N. M., Ahmed, A. S., and Ebraheim, Z. Z. (2009). Antimicrobial effects of pepper, parsley, and dill and their roles in the microbiological quality enhancement of traditional Egyptian Kareish cheese. Foodborne Pathog. Dis. 7, 411-418. doi: 10.1089/fpd.2009.0412

Wang, Q., Garrity, G. M., Tiedje, J. M., and Cole, J. R. (2007). Naive Bayesian classifier for rapid assignment of rRNA sequences into the new bacterial taxonomy. Appl. Environ. Microbiol. 73, 5261-5267. doi: 10.1128/AEM.00062-07

Wang, C., Liao, M., Zhang, Z., and Wu, Z. (2017). The influence of three Chinese medicinal herbs on the growth and antioxidant properties of lactic acid bacteria. Food Ind. 7, 32-35.

Wu, D. T., Fu, Y., Guo, H., Yuan, Q., Nie, X. R., Wang, S. P., et al. (2021a). In vitro simulated digestion and fecal fermentation of polysaccharides from loquat leaves: dynamic changes in physicochemical properties and impacts on human gut microbiota. Int. J. Biol. Macromol. 168, 733-742. doi: 10.1016/j. ijbiomac.2020.11.130

Wu, D., Nie, X., Gan, R., Guo, H., Fu, Y., Yuan, Q., et al. (2021c). In vitro digestion and fecal fermentation behaviors of a pectic polysaccharide from okra (Abelmoschus esculentus) and its impacts on human gut microbiota. Food Hydrocoll. 114:106577. doi: 10.1016/j.foodhyd.2020.106577

Wu, D. T., Yuan, Q., Guo, H., Fu, Y., Li, F., Wang, S. P., et al. (2021b). Dynamic changes of structural characteristics of snow chrysanthemum polysaccharides during in vitro digestion and fecal fermentation and related impacts on gut microbiota. Food Res. Int. 141:109888. doi: 10.1016/j. foodres.2020.109888

Xiong, T., Guan, Q., Song, S., Hao, M., and Xie, M. (2012). Dynamic changes of lactic acid bacteria flora during chinese sauerkraut fermentation. Food Control 26, 178-181. doi: 10.1016/j.foodcont.2012.01.027

Xiong, T., Li, J., Liang, F., Wang, Y., Guan, Q., and Xie, M. (2016). Effects of salt concentration on chinese sauerkraut fermentation. LWT Food Sci. Technol. 69, 169-174. doi: 10.1016/j.lwt.2015.12.057

Yang, X., Hu, W., Xiu, Z., Jiang, A., Yang, X., Saren, G., et al. (2020). Microbial community dynamics and metabolome changes during spontaneous fermentation of northeast sauerkraut from different households. Front. Microbiol. 11:1878. doi: 10.3389/fmicb.2020.01878

Yu, Y., Lee, C., Kim, J., and Hwang, S. (2005). Group-specific primer and probe sets to detect methanogenic communities using quantitative real-time 
polymerase chain reaction. Biotechnol. Bioeng. 89, 670-679. doi: 10.1002/ bit.20347

Yu, Z., Zhang, X., Li, S., Li, C., Li, D., and Yang, Z. (2013). Evaluation of probiotic properties of Lactobacillus plantarum strains isolated from chinese sauerkraut. World J. Microbiol. Biotechnol. 29, 489-498. doi: 10.1007/ s11274-012-1202-3

Zabat, M. A., Sano, W. H., Wurster, J. I., Cabral, D. J., and Belenky, P. (2018). Microbial community analysis of sauerkraut fermentation reveals a stable and rapidly established community. Foods 7:77. doi: 10.3390/foods7050077

Zanirati, D. F., Abatemarco, M., Sandes, S. H. C., Nicoli, J. R., Nunes, Á. C., and Neumann, E. (2015). Selection of lactic acid bacteria from Brazilian kefir grains for potential use as starter or probiotic cultures. Anaerobe 32, 70-76. doi: 10.1016/j.anaerobe.2014.12.007

Zhou, X., Zhang, Z., Huang, F., Yang, C., and Huang, Q. (2020). In vitro digestion and fermentation by human fecal microbiota of polysaccharides from flaxseed. Molecules 25:4354. doi: 10.3390/molecules25194354
Conflict of Interest: The authors declare that the research was conducted in the absence of any commercial or financial relationships that could be construed as a potential conflict of interest.

Publisher's Note: All claims expressed in this article are solely those of the authors and do not necessarily represent those of their affiliated organizations, or those of the publisher, the editors and the reviewers. Any product that may be evaluated in this article, or claim that may be made by its manufacturer, is not guaranteed or endorsed by the publisher.

Copyright (c) 2021 Chan, Liu, Wu, Yang and Howell. This is an open-access article distributed under the terms of the Creative Commons Attribution License (CC BY). The use, distribution or reproduction in other forums is permitted, provided the original author(s) and the copyright owner(s) are credited and that the original publication in this journal is cited, in accordance with accepted academic practice. No use, distribution or reproduction is permitted which does not comply with these terms. 